\title{
Reporting Rules in Bank Runs
}

\author{
July 2021
}

\begin{abstract}
We study the role of reporting rules in the context of bank runs. In our model, a financial institution receives an early but imprecise estimate of the performance of its investment and issues a report subject to a reporting rule. We find that, from a financial-stability standpoint, the optimal reporting rule requires full disclosure when the financial institution's early estimate is sufficiently unfavorable, but no disclosure otherwise. Importantly, the threshold below which the financial institution reports should be tailored to the financial institution's exposure to bank-run risk. In particular, the optimal reporting threshold is non-monotonic and U-shaped in the bank-run risk. We also relate our results to current accounting standards for asset impairments.
\end{abstract}

Keywords: Reporting rules; impairment; financial institutions; bank runs; financial stability.

JEL Codes: G21, G28, M41, M48. 


\section{Introduction}

Fair value accounting rules are often blamed for contributing to financial instability. One most notable instance is during the 2007-2009 financial crisis, many critics attributed fair value accounting as a cause for the financial meltdown and the panic-based runs on financial institutions. ${ }^{1}$ However, it is probably less recognized that most of financial institutions' assets are not reported at fair value in its purest form, but instead at a mixture of historical cost and fair value (Laux and Leuz, 2010). For example, loans and leases, which account for a majority of these institutions' assets, are initially measured at historical cost and later subject to impairment testing, depending on their fair value: "An investment is impaired if its fair value is less than its cost." (Financial Accounting Standards Board, ASC 320-10-35-21).

This paper seeks to understand the role of reporting rules in maintaining financial stability. In a canonical bank-run model (Morris and Shin, 2000), we show that the optimal reporting rule resembles some aspects of the impairment rules in current accounting standards as it requires full disclosure of unfavorable news but no disclosure of favorable news. This result thus implies that, in contrast to the criticism of fair value measurement for destabilizing financial markets, the actual implementation of fair value measurement based on impairment testing may play a stabilizing role. We also characterize the optimal threshold below which financial institutions should report, which generates implications for

\footnotetext{
${ }^{1}$ As a perhaps more extreme example, Steve Forbes, chairman of Forbes Media, alleged that mark-to-market accounting was "the principal reason" that the U.S. financial system melted down. See https://hbr.org/2009/11/is-it-fair-to-blame-fair-value-accounting-for-the-financial-crisis. The 2007-2009 financial turmoil also reveals ample evidence on the fragility of financial institutions to risk of runs, especially in the "shadow banking" markets that are not covered within traditional government safety nets such as the federal deposit insurance. See Brunnermeier (2009), Shin (2009), and Acharya, Schnabl, and Suarez (2013) for examples of runs in repo markets, runs on commercial banks such as Northern Rock, and runs in the asset-backed commercial papers market, respectively.
} 
how impairment tests should be conducted in practice. We show that, from a financialstability standpoint, the reporting threshold should be tailored to financial institutions' exposure to bank-run risk.

We consider a model that consists of a representative financial institution that finances its project (e.g., a loan) by attracting investments from a group of investors. The financial institution is vulnerable to runs because its investors can withdraw their investments even before the return from the institution's project is realized. Such early withdrawals are costly as they force the financial institution to liquidate its project prematurely to meet the withdrawal requests, thus resulting in liquidation losses. Prior to the investors' withdrawal decisions, the financial institution observes an early but imprecise estimate of its project return and issues a report about the project return subject to some reporting rules. We do not restrict the form of the reporting rules but instead focus on characterizing the optimal rule that maximizes the total surplus. Upon receiving the report, the investors then use it in conjunction with their own private information in determining whether to withdraw from the financial institution.

We first examine a benchmark in which the financial institution discloses its early estimate fully. We find that while full disclosure eliminates inefficient continuation of insolvent financial institutions, this does not achieve full efficiency as it sustains inefficient runs on solvent financial institutions. These runs are panic-based and triggered by investors' strategic uncertainty about other investors' decisions to withdraw.

Our analysis next characterizes the optimal reporting rule, which exhibits two salient features. First, the optimal reporting rule mandates full disclosure only when the financial 
institution's early estimate of its investment outcome falls below a threshold; otherwise, the financial institution should not recognize the estimate in its report. This reporting rule is consistent with the impairment rule under current accounting standards and thus lends some support to the social value of the impairment rule in maintaining financial stability. Second, the optimal reporting rule ensures no runs on the set of financial institutions that do not disclose, even though continuing those institutions may sometimes be inefficient. Note that this feature of the optimal rule stands in stark contrast to that of full disclosure. While full disclosure avoids all inefficient continuations at the expense of sustaining inefficient runs, the optimal rule aims at achieving a better balance between the two types of inefficiency, tolerating some inefficient continuations in exchange for fewer inefficient runs.

We further explore how the optimal reporting threshold should be set. Our main result is that, from a financial-stability standpoint, the reporting threshold should be tailored to the magnitude of the bank-run risk that the financial institution faces. In particular, the optimal reporting threshold is actually non-monotonic and U-shaped in the bank-run risk. The intuition is as follows. When the bank-run risk increases from a relatively low level, it is optimal to lower the reporting threshold and reveal less unfavorable news to mitigate the increase of inefficient runs. However, when the bank-run risk rises above a certain cutoff, the reporting threshold should be raised to disclose more bad news. This adjustment is optimal under the circumstances of heightened bank-run risk as it improves investors' assessment of the quality of the financial institutions in the no-disclosure set such that these institutions continue to suffer no runs. 


\subsection{Related Literature}

This paper is related to the accounting and economics literature on the role of information disclosure in bank runs. Financial institutions, such as commercial banks, investment banks, and investment funds, are exposed to the risk of runs due to the liquidity and duration mismatch between their assets and liabilities (Diamond and Dybvig, 1983). Opacity is often attributed as a key contributor to financial instability (Morgan, 2002). Toward that end, the seminal paper by Morris and Shin (2000) develops a tractable framework of bank runs suitable for examining the role of information disclosure in bank runs, which has then been widely employed in the literature. Prior papers have mostly focused on examining specific information structure in a Morris-Shin setting, and thus do not directly address the issue of ex ante optimal reporting rules, which is a key focus of our paper. For instance, Bouvard, Chaigneau, and Motta (2015) consider a setting with a continuum of banks, whose fundamental consists of a uniformly-distributed aggregate component and a binary idiosyncratic component. A regulator chooses to either disclose the idiosyncratic components of all banks fully or none. Their main result is that the regulator should disclose if the aggregate shock is sufficiently bad. Gao and Jiang (2018) examine the role of banks' ex post reporting discretion in affecting runs. Liang and Zhang (2021) employ a structure of correlated private information to represent the classic accounting trade-off of objectivity and accuracy and study the value of accounting objectivity in mitigating inefficient runs. Our paper contributes to this literature by characterizing the optimal reporting rule within the Morris-Shin framework. The optimal rule we derive is consistent with the impairment rule in accounting standards and thus lends some support to adopting the impairment rule on 
the grounds of financial stability.

This paper is also related to the literature on the information environment of financial institutions. This literature is vast and thoroughly reviewed in three surveys by Beatty and Liao (2014), Goldstein and Sapra (2014), and Acharya and Ryan (2016). Some recent papers have focused on examining the use of accounting information in the prudential regulation of banks (e.g., Corona, Nan and Zhang, 2015, 2019a, 2019b; Mahieux, Sapra and Zhang, 2020; Bertomeu, Mahieux and Sapra, 2021). Perhaps more related to our paper, Goldstein and Leitner (2018) consider a model in which disclosure may hinder risk-sharing among banks but under other circumstances, some partial disclosure is necessary for risk sharing to occur. In contrast to our paper, the optimal disclosure rule in Goldstein and Leitner (2018) is inconsistent with the impairment rule as it can take a more complicated form (e.g., multiple cutoffs or non-monotone rules).

More broadly, this paper is related to the extensive disclosure literature. Due to the size of this literature, we refer interested readers to several recent reviews by Beyer et al. (2010), Ewert and Wagenhofer (2012), Stocken (2013), and Armstrong et al. (2016). ${ }^{2}$ Mostly related to our paper, Göx and Wagenhofer (2009) examine the optimal accounting policy in a setting in which a financially-constrained firm raises debts from a lender. The key friction in their setting is that the firm's management may lack incentive to exert effort, which creates a need for the firm to pledge its assets to the lender to mitigate the moral hazard problem. ${ }^{3}$ Accordingly, the measurement rule of the asset value plays an essential role in

\footnotetext{
${ }^{2}$ In particular, recent accounting literature studies ex ante information design applying the framework of Bayesian persuasion following Kamenica and Gentzkow (2011), for example, Jiang and Yang (2017), Huang (2019), Friedman et al. (2020, 2021), and Laux and Zheng (2021).

${ }^{3}$ More recently, Bertomeu and Cheynel (2015) and Jiang and Yang (2021) also study the optimal measurement rules considering frictions of both inefficient continuation and liquidation in a setting of
} 


\begin{tabular}{|c|c|c|c|}
\hline$t=0$ & $t=1$ & $t=2$ & $t=3$ \\
\hline $\begin{array}{l}\text { A reporting rule is } \\
\text { prescribed. FI finances from } \\
\text { investors and invests. }\end{array}$ & $\begin{array}{l}\text { FI learns information } \lambda \text {, } \\
\text { and issues report } x \text { according } \\
\text { to reporting rule. }\end{array}$ & $\begin{array}{l}\text { Investors learn private } \\
\text { signal } s_{i} \text { and decide } \\
\text { whether to withdraw. }\end{array}$ & $\begin{array}{l}\text { Investment outcome } \\
\text { is realized. }\end{array}$ \\
\hline
\end{tabular}

Figure 1: Timeline of the model.

determining whether the firm's project can be successfully financed. Similar to our paper, Göx and Wagenhofer also find that the impairment rule is optimal in the debt-financing setting. In this light, our paper shows that the optimality of the impairment rule extends to a bank-run setting that entails different frictions (i.e., panic-based runs and inefficient continuations) from the moral-hazard issue in the debt-financing setting. Our analysis also generates implications regarding how the impairment threshold should be set contingent on the level of bank-run risk, which Göx and Wagenhofer do not study.

The rest of the paper proceeds as follows. Section 2 describes our model. Section 3 analyzes the model. Section 4 discusses some extensions of the model and Section 5 concludes. An Appendix contains the proofs of our results.

\section{The Model}

We examine an environment that consists of a representative financial institution (henceforth, FI) and a continuum of investors. Figure 1 summarizes the timing of events.

At $t=0$, the FI is endowed with an investment project that yields a stochastic gross rate of return $\tilde{R}=e^{\tilde{r}} \geq 0$ realized at $t=3$. The FI finances the project by attracting investments from a group of investors, with unit mass indexed by the unit interval $[0,1]$, corporate finance related to Göx and Wagenhofer (2009). 
each of whom contributes 1 unit of the consumption good. ${ }^{4}$ All investors have the log utility function $u_{i}=\log \left(c_{i 2}+c_{i 3}\right)$, where $c_{i 2}$ and $c_{i 3}$ denote investor $i$ 's consumption at $t=2$ and at $t=3$ respectively. The FI invests all the investments attracted in its project.

The investment return depends on two independent random variables $\tilde{\lambda}$ and $\tilde{\theta}$ such that

$$
\tilde{r}=\tilde{\lambda}+\tilde{\theta}
$$

$\tilde{\lambda}$ has a general distribution $F($.$) and a density f($.$) with full support on [-\bar{\lambda}, \bar{\lambda}] . \tilde{\theta}$ follows a uniform distribution in the interval $[-\bar{\theta}, \bar{\theta}]$. In Section 4.1, we relax the assumption of uniform distribution and consider a general distribution for $\tilde{\theta}$. The FI observes $\lambda$ at $t=1$, which represents some early, albeit imperfect, information about the investment return before the return is realized. The random variable $\tilde{\theta}$ thus captures the residual uncertainty regarding the investment return after the FI learns $\lambda$. This dichotomy captures the property of accounting systems in which not all information is amenable to inclusion in accounting reports (e.g., Ijiri, 1975).

At $t=0$, an ex ante reporting rule is prescribed and governs how the FI issues a report $x$ about $\lambda$ at $t=1$. We do not place any restrictions on how the reporting rule is set, but rather derive the optimal reporting rule that maximizes the expected total surplus. For instance, the reporting rule may be perfectly informative about all $\lambda$, partially informative about only some values of $\lambda$, or not informative at all. For another example, the reporting rule may take the form of an impairment rule that reveals information about $\lambda$ only when $\lambda$

\footnotetext{
${ }^{4}$ Note that investors always choose to fund the FI at $t=0$ because, as we will discuss later, they are allowed to redeem their original investments at the face value at $t=2$.
} 
is sufficiently low (Göx and Wagenhofer, 2009). ${ }^{5}$

At $t=1$, the FI observes the early information $\lambda$ and issues a report $x$ about $\lambda$ according to the reporting rule. At $t=2$, along with the FI's report $x$, each investor $i$ observes a private signal $s_{i}$ about the random variable $\tilde{\theta}$ :

$$
s_{i}=\tilde{\theta}+\tilde{\varepsilon}_{i}
$$

where $\tilde{\varepsilon}_{i} \sim N\left(0, \frac{1}{\beta}\right) .{ }^{6}$ Following the global games literature (Morris and Shin, 2000; Gao and Jiang, 2018), we consider in our main analysis a limiting case in which $\beta$ approaches infinity such that each investor observes $\theta$ (almost) perfectly. ${ }^{7}$ We resort to the global games technique to obtain a unique equilibrium outcome in bankrun models that would otherwise feature multiple equilibria, and the equilibrium uniqueness is instrumental in deriving the optimal reporting rule. ${ }^{8}$

Given her information set $\left\{x, s_{i}\right\}$, investor $i$ decides whether to withdraw her investment. We model the investors' withdrawal decisions following the bankrun models in Morris and

\footnotetext{
${ }^{5}$ It is noteworthy that financial institutions often apply some forms of impairment rules to measure their assets, especially held-to-maturity (HTM) debt securities such as loans (ASC 320). In particular, financial institutions must evaluate whether to report losses when the fair value of their HTM assets is below the amortization cost. However, financial institutions are usually not allowed to report gains when the fair value exceeds the amortization cost.

${ }^{6}$ Our assumption that investors are equipped with private information is consistent with the characteristics of investors participating in the modern-day run on financial institutions in the shadow banking market. These investors are primarily sophisticated institutional investors, such as mutual funds, investment banks, and hedge funds. Different from ordinary investors, these investors are trained professionals, well equipped with private information about operations in shadow banking markets.

${ }^{7}$ In Section 4.2, we consider an extension in which the investors observe $\tilde{\theta}$ with some noise. Consistent with the results in the global games literature (e.g., Morris and Shin, 2000; Liang and Zhang, 2019), when the precision of private information is sufficiently high, we obtain a unique equilibrium outcome in the bankrun subgame and all the results in our main setting hold qualitatively.

${ }^{8}$ The global games technique has been widely used in coordination games that feature multiple equilibria, including bankrun models, to obtain uniqueness. The equilibrium selection made by the global games approach is supported by evidence in numerous experimental studies (Anctil et al., 2004; Heinemann, Nagel, and Ockenfels, 2004; Cabrales, Nagel, and Armenter, 2007; Anctil et al., 2010).
} 
Shin (2000), Gao and Jiang (2018) and Liang and Zhang (2019). If investor $i$ withdraws, she is repaid at the face value, 1 unit of the consumption good. Further, the FI's project is illiquid and the net rate of return obtainable at $t=3$ is decreasing in the proportion of investments withdrawn at $t=2$, as denoted by $l \in[0,1]$. Specifically, we assume that at $t=3$, the net rate of return is $e^{\tilde{r}-\delta l}$. The term $e^{-\delta l}<1$ represents the cost of liquidating the illiquid project to meet the investors' withdrawals. The parameter $\delta>0$ captures the extent to which the withdrawals impair the investment return. At $t=3$, the net rate of return is realized and distributed to the investors.

Finally, to make the problem interesting, we impose the following assumption:

Assumption 1: Conditional on the most favorable report $x=\bar{\lambda}$, no investors will withdraw from the FI. As it turns out, this assumption reduces into

$$
\bar{\lambda}>\bar{\theta}+\frac{\delta}{2} .
$$

Assumption 1 ensures that there exists a reporting rule that can at least stabilize runs in some cases. Otherwise, runs can always occur regardless of the reporting rule.

\section{Analysis}

\subsection{The bankrun equilibrium}

We solve the model by backward induction and derive first the investors' withdrawal decisions at $t=2$. As shown in Morris and Shin (2000), it is without loss of generality to consider only the switching strategy in which an investor chooses to withdraw if and only if her 
private signal $s_{i}$ is below some threshold $\hat{s}$. To derive the equilibrium threshold $\hat{s}$, consider a marginal investor whose signal $s_{i}$ is exactly equal to $\hat{s}$. If she withdraws, she receives 1 unit of consumption goods and obtains a utility of $\log 1=0$. If she chooses not to withdraw, her expected utility is $\mathbb{E}\left[\log \left(e^{\tilde{r}-\delta l}\right) \mid \hat{s}, x\right]=\mathbb{E}[\tilde{r}-\delta l \mid \hat{s}, x]$, which is equal to

$$
\mathbb{E}[r \mid x, \hat{s}]-\delta \mathbb{E}[l \mid x, \hat{s}]=\mathbb{E}[\lambda \mid x]+\mathbb{E}[\theta-\delta \Phi(\sqrt{\beta}(\hat{s}-\theta)) \mid \hat{s}]
$$

$\Phi(\cdot)$ stands for the cumulative standard normal distribution function. To understand (4), note that the investor's updated belief of $\tilde{r}$ conditional upon her information set $\{x, \hat{s}\}$ is $\mathbb{E}[r \mid x, \hat{s}]=\mathbb{E}[\lambda \mid x]+\mathbb{E}[\theta \mid \hat{s}]$. In addition, by the law of large numbers, the marginal investor's expectation of the portion of investors who withdraw $\mathbb{E}[l \mid x, \hat{s}]$ is equal to the probability that a particular investor $j$ withdraws. Because investor $j$ also follows the same switching strategy, she withdraws if and only if her signal $s_{j} \leqslant \hat{s}$, i.e.,

$$
\mathbb{E}[l \mid x, \hat{s}]=\mathbb{E}\left[\operatorname{Pr}\left(s_{j} \leqslant \hat{s} \mid \theta\right) \mid \hat{s}\right]=\mathbb{E}[\Phi(\sqrt{\beta}(\hat{s}-\theta)) \mid \hat{s}]
$$

In equilibrium, the investor who observes a $s_{i}$ equal to $\hat{s}$ is indifferent between staying and withdrawing. This in turn gives:

$$
\mathbb{E}[\lambda \mid x]+\mathbb{E}[\theta-\delta \Phi(\sqrt{\beta}(\hat{s}-\theta)) \mid \hat{s}]=0
$$


When $\beta$ approaches infinity, the private signal $\hat{s}$ approaches $\theta$, and (6) gives $\hat{s}=\frac{\delta}{2}-\mathbb{E}[\lambda \mid x]$. Therefore, an investor withdraws if and only if

$$
s_{i}=\theta \leq \hat{s}=\frac{\delta}{2}-\mathbb{E}[\lambda \mid x]
$$

We summarize the investors' equilibrium decisions in the following lemma.

Lemma 1 Investors withdraw from the FI if and only if $\mathbb{E}[\lambda \mid x]+\theta \leq \frac{\delta}{2}$.

An important message from Lemma 1 is that the investors' withdrawal decisions in equilibrium may not necessarily be socially optimal. To elaborate, we first derive the socially optimal withdrawal decision that maximizes the total surplus. Given that the FI always breaks even, the total surplus is equal to the sum of all investors' utilities, $\int_{0}^{1} u_{i} d i$. The total surplus is $\int_{0}^{1} \log (1) d i=0$ if the investors withdraw, whereas it is $\int_{0}^{1} \log \left(e^{\lambda+\theta}\right) d i=\lambda+\theta$ if the investors stay. It is therefore socially optimal to withdraw from the FI if and only if $\lambda+\theta \leq 0$. Note that, compared to the socially optimal decisions, the investors may withdraw either too much or too little. For our convenience, we define the two types of inefficiency as follows.

Definition 1 Inefficient run occurs when $\lambda+\theta>0$ and investors withdraw, whereas inefficient continuation occurs when $\lambda+\theta<0$ and investors stay.

\subsection{Optimal reporting rule}

We now turn to our main research question of, from a financial-stability perspective, how the reporting rules should be set optimally to maximize the social surplus. Lemma 1 
suggests that the optimal reporting rules must jointly minimize both inefficient runs on the FI and inefficient continuations of the FI. Before we characterize the optimal reporting rules, it is instructive to examine whether full disclosure achieves full efficiency. The following proposition shows that this claim does not hold in general.

Proposition 1 Under full disclosure, i.e., $x=\lambda$, the equilibrium is as follows:

1. if $\lambda \geq \bar{\theta}+\frac{\delta}{2}$, no investors withdraw and the equilibrium is efficient;

2. if $\lambda \in\left[\bar{\theta}, \bar{\theta}+\frac{\delta}{2}\right)$, investors withdraw when $\theta<-\lambda+\frac{\delta}{2}$ and inefficient run occurs when $\theta \in\left(-\bar{\theta},-\lambda+\frac{\delta}{2}\right)$

3. if $\lambda \in\left[-\bar{\theta}+\frac{\delta}{2}, \bar{\theta}\right)$, investors withdraw when $\theta<-\lambda+\frac{\delta}{2}$ and inefficient run occurs when $\theta \in\left(-\lambda,-\lambda+\frac{\delta}{2}\right)$

4. if $\lambda \in\left[-\bar{\theta},-\bar{\theta}+\frac{\delta}{2}\right)$, investors always withdraw and inefficient run occurs when $\theta \in$ $(-\lambda, \bar{\theta})$

5. if $\lambda<-\bar{\theta}$, investors always withdraw and the equilibrium is efficient.

Proposition 1 highlights both the benefit and the cost of implementing the full disclosure rule. As shown in Figure 2, while full disclosure eliminates inefficient continuation, it does so at the expense of sustaining some inefficient runs when the disclosure $\lambda$ is of some intermediate value. The reason is that, with full disclosure, the investors withdraw from the FI excessively relative to what is socially optimal. Fearing that others may withdraw, each individual investor may also choose to withdraw even if the continuation payoff of the project, absent withdrawals, exceeds the liquidation payoff. Such inefficient "panic-based 


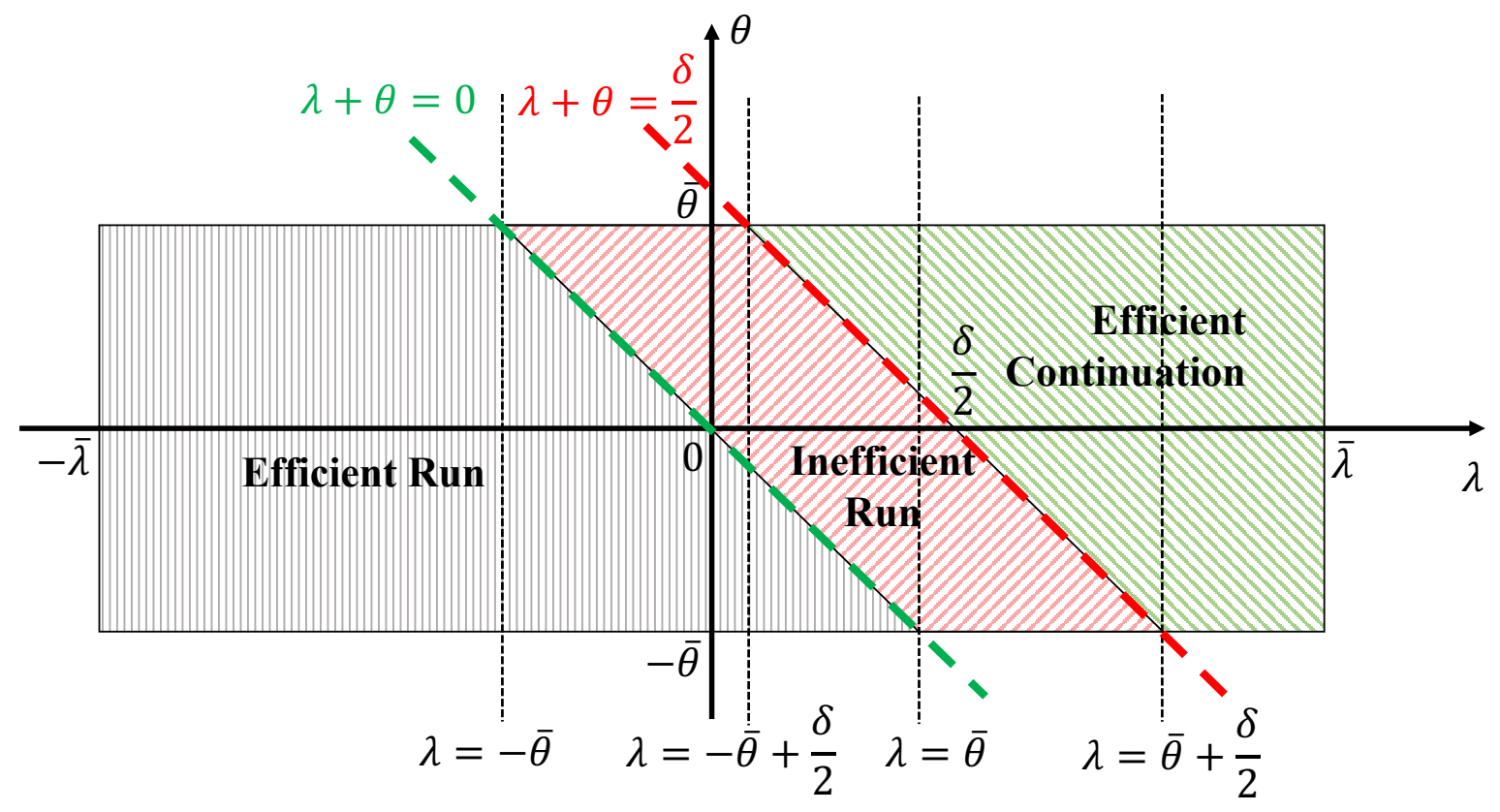

Figure 2: An illustration of Proposition 1.

runs" indeed become self-fulfilling in equilibrium when the FI's disclosure is moderate such that the coordination among the investors matters the most.

An implication of Proposition 1 is that, it may be surplus-enhancing to tolerate some inefficient continuations in exchange for fewer inefficient runs. Apparently, this move requires a departure from full disclosure. Toward that end, we characterize in Propositions 2 and 3 some properties of the partial-disclosure rules that aim at achieving a better balance between inefficient runs and inefficient continuations. Proposition 2 first provides a set of necessary conditions for the optimal reporting rule.

Proposition 2 The optimal reporting rule must satisfy the following necessary conditions:

1. if $\lambda<-\bar{\theta}$, the FI shall always report $\lambda$ perfectly;

2. if $\lambda>\bar{\theta}+\frac{\delta}{2}$, the FI shall always disclose the same report $x_{H}$; 
3. if $\lambda \in\left[-\bar{\theta}, \bar{\theta}+\frac{\delta}{2}\right]$, the FI shall either report $x_{H}$ or report $\lambda$ perfectly.

The first two parts of Proposition 2 are intuitive. Part 1 states that it is never optimal to withhold any information when $\lambda<-\bar{\theta}$. The reason is that, when $\lambda<-\bar{\theta}$, the liquidation payoff of the FI's project always exceeds the continuation payoff for any value of $\theta$ (i.e., $\lambda+\theta<0)$ so withdrawing from the FI is the socially optimal decision. From Lemma 1, disclosing that $\lambda<-\bar{\theta}$ indeed triggers withdrawals by the investors, thus achieving efficiency. Part 2 of Proposition 2 states that it is optimal to pool all FIs with $\lambda>\bar{\theta}+\frac{\delta}{2}$ together. This is because the investors will never withdraw inefficiently from the group of FIs with high $\lambda$, regardless of whether they disclose fully or are pooled. Furthermore, pooling yields an additional benefit as it allows some FIs that receive a lower $\lambda \in\left[-\bar{\theta}, \bar{\theta}+\frac{\delta}{2}\right]$ to be mixed with the group of FIs with $\lambda>\bar{\theta}+\frac{\delta}{2}$. This improves the investors' assessment of $\lambda$ for the pooled FIs with $\lambda \in\left[-\bar{\theta}, \bar{\theta}+\frac{\delta}{2}\right]$, which helps to avoid inefficient runs on them.

Part 3 of Proposition 2 merits more discussion. It states that the FIs with $\lambda \in\left[-\bar{\theta}, \bar{\theta}+\frac{\delta}{2}\right]$ shall either be pooled with the FIs with $\lambda>\bar{\theta}+\frac{\delta}{2}$ (via reporting the same $x_{H}$ ) or fully separated from others (via full disclosure), but never pooled among themselves. This result reflects a consideration to optimally mitigate runs across different values of $\lambda \in\left[-\bar{\theta}, \bar{\theta}+\frac{\delta}{2}\right]$. To elaborate, consider a simple example with two FIs each of whom learns $\lambda=\lambda_{1}$ and $\lambda=\lambda_{2}$, respectively, and $-\bar{\theta}<\lambda_{2}<\lambda_{1}<\bar{\theta}+\frac{\delta}{2}$, i.e., FI- $\lambda_{2}$ is financially weaker than FI- $\lambda_{1}$. We compare two cases, a separating case in which the two FIs issue different reports, and a pooling case in which two FIs issue the same report. Figure 3 suggests that, relative to separating, pooling with the stronger FI- $\lambda_{1}$ helps to reduce runs on the weaker FI- $\lambda_{2}$ because, under pooling, the FI- $\lambda_{2}$ will no longer be liquidated if $\theta \in\left[\frac{\delta}{2}-\frac{\lambda_{1}+\lambda_{2}}{2}, \frac{\delta}{2}-\lambda_{2}\right)$. However, 


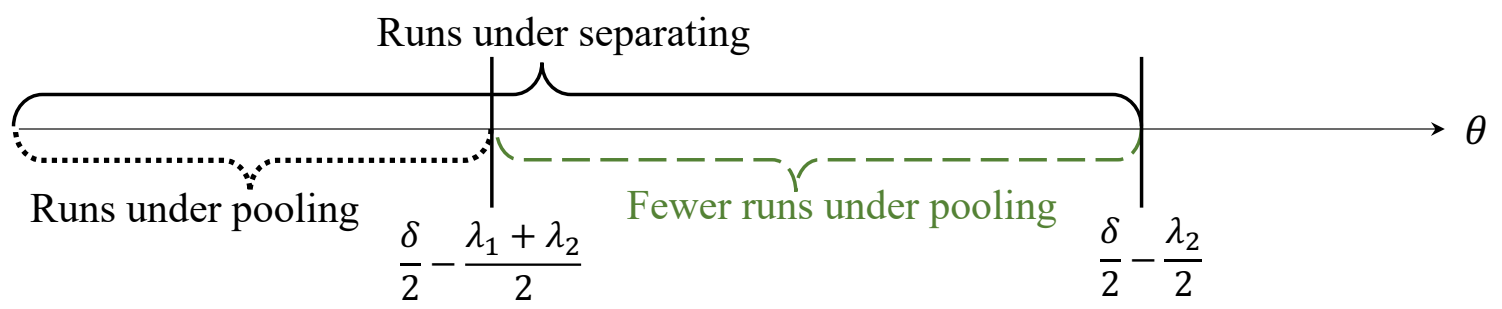

Panel A: bank run equilibrium for FI- $\lambda_{2}$.

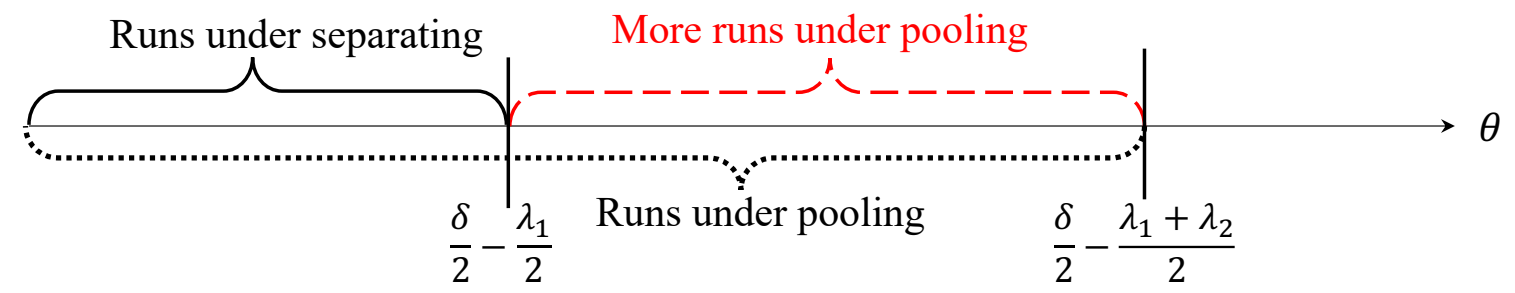

Panel B: bank run equilibrium for FI- $\lambda_{1}$.

Figure 3: An illustration of Proposition 2. In Panel A, when $\lambda_{2}$ is disclosed, the investors withdraw if and only if $\theta<\frac{\delta}{2}-\lambda_{2}$, while when the two FIs are pooled, $\mathbb{E}[\lambda \mid x]=\frac{\lambda_{1}+\lambda_{2}}{2}$, and the investors withdraw if and only if $\theta<\frac{\delta}{2}-\frac{\lambda_{1}+\lambda_{2}}{2}$ (Lemma 1). Therefore, the FI- $\lambda_{2}$ is no longer liquidated when $\theta \in\left[\frac{\delta}{2}-\frac{\lambda_{1}+\lambda_{2}}{2}, \frac{\delta}{2}-\lambda_{2}\right)$ under pooling. Similarly in Panel B, when $\lambda_{1}$ is disclosed, the investors withdraw if and only if $\theta<\frac{\delta}{2}-\lambda_{1}$. The FI- $\lambda_{1}$, therefore, is liquidated more often when $\theta \in\left[\frac{\delta}{2}-\lambda_{1}, \frac{\delta}{2}-\frac{\lambda_{1}+\lambda_{2}}{2}\right)$ under pooling. 
pooling also triggers more runs on the stronger FI- $\lambda_{1}$ when $\theta \in\left[\frac{\delta}{2}-\lambda_{1}, \frac{\delta}{2}-\frac{\lambda_{1}+\lambda_{2}}{2}\right)$. In other words, pooling essentially substitutes runs on weak FIs with runs on strong ones. Note that under an optimal reporting rule that maximizes the total surplus, one should never make such substitution because, ceteris paribus, it is never worth saving the less valuable weak FIs (e.g., FI- $\lambda_{2}$ ) at the expense of failing the more valuable strong FIs (e.g., FI- $\left.\lambda_{1}\right) .{ }^{9}$ This explains why the FIs with $\lambda \in\left[-\bar{\theta}, \bar{\theta}+\frac{\delta}{2}\right]$ should not be pooled among themselves.

A key message of Proposition 2 is that the optimal reporting rule is a dichotomy that mandates full disclosure at some values of $\lambda$ but a report insensitive to $\lambda$ elsewhere. Relative to full disclosure, the partial-pooling rule can be desirable because mixing weak FIs with stronger ones helps to reduce runs on the weak FIs. However, this benefit of pooling is limited by a downside. Intuitively, as more weak FIs are added to the pooling group, it can lower investors' assessment of the quality of the pooled FIs and thus put those pooled FIs at higher risk for suffering runs. Proposition 3 shows that this downside is a dominant consideration. In fact, weak FIs should be added only when this does not trigger any runs on the pooled FIs.

Proposition 3 Under the optimal reporting rule, the investors never withdraw from the pooling group that reports $x_{H}$ for any realization of $\theta$, i.e., $\mathbb{E}\left(\lambda \mid x_{H}\right) \geq \bar{\theta}+\frac{\delta}{2}$.

The intuition for Proposition 3 can be gleaned similarly as in part 3 of Proposition 2. Since the surplus generated from the pooled high- $\lambda$ FIs are always greater than that from

\footnotetext{
${ }^{9}$ Mathematically, the expected surplus loss from pooling is $E L \equiv \frac{1}{2 \bar{\theta}} \int_{\frac{\delta}{2}-\lambda_{1}}^{\frac{\delta}{2}-\frac{\lambda_{1}+\lambda_{2}}{2}}\left(\lambda_{1}+\theta\right) d \theta$ whereas the expected gain from pooling is $E G \equiv \frac{1}{2 \theta} \int_{\frac{\delta}{2}-\frac{\lambda_{1}+\lambda_{2}}{2}}^{\frac{\delta}{2}-\lambda_{2}}\left(\lambda_{2}+\theta\right) d \theta$. Note that the surplus loss always outweighs the surplus gain, i.e., $E L-E G=\frac{\left(\lambda_{1}-\lambda_{2}\right)^{2}}{8 \bar{\theta}}>0$.
} 
the low- $\lambda$ FIs to be added, it hurts the total surplus to trade runs on the weak FIs for runs on the strong ones. This no-run-on-pooled-FIs requirement naturally sets a bound on the mass of weak FIs that can be included in the pooling group.

The final step toward deriving the optimal reporting rule is to determine which of the FIs with moderate $\lambda \in\left[-\bar{\theta}, \bar{\theta}+\frac{\delta}{2}\right]$ shall be pooled with the strong FIs with $\lambda>\bar{\theta}+\frac{\delta}{2}$, while respecting the pooling constraint outlined in Proposition 3. Denote by $\Lambda$ the set of the FIs that learn $\lambda \in\left[-\bar{\theta}, \bar{\theta}+\frac{\delta}{2}\right]$ but are pooled with the FIs with $\lambda>\bar{\theta}+\frac{\delta}{2}$. The optimal $\Lambda$ is set by solving the following program: ${ }^{10}$

$$
\begin{aligned}
& \max _{\Lambda \subseteq\left[-\bar{\theta}, \frac{\delta}{2}+\bar{\theta}\right]} \int_{\lambda \in \Lambda \cup\left(\frac{\delta}{2}+\bar{\theta}, \bar{\lambda}\right]} \lambda f(\lambda) d \lambda+\int_{\lambda \in\left[-\bar{\theta}, \frac{\delta}{2}+\bar{\theta}\right] \backslash \Lambda} W(\lambda) f(\lambda) d \lambda, \\
& \text { s.t., } \mathbb{E}\left[\lambda \mid \lambda \in \Lambda \cup\left[\frac{\delta}{2}+\bar{\theta}, \bar{\lambda}\right]\right] \geq \frac{\delta}{2}+\bar{\theta} .
\end{aligned}
$$

The constraint of the optimization program stems from the requirement of Proposition 3 that runs on the pooling group must be avoided. The objective function of the program reflects the consequences of pooling. For the set of FIs that are included in the pooling group (i.e., $\left.\lambda \in \Lambda \cup\left(\frac{\delta}{2}+\bar{\theta}, \bar{\lambda}\right]\right)$, they never experience runs for any realization of $\theta$ (Proposition 3). For the set of FIs that are excluded (i.e., $\lambda \in\left[-\bar{\theta}, \frac{\delta}{2}+\bar{\theta}\right] \backslash \Lambda$ ), they are required to disclose fully and thus survive runs only upon favorable realizations of $\theta>\frac{\delta}{2}-\lambda$ (Part 3 of Proposition 2). A pooled FI's project generates an expected surplus of $\mathbb{E}[\lambda+\theta]=\lambda$ whereas a non-pooled

\footnotetext{
${ }^{10}$ The notation $\Lambda \cup\left[\frac{\delta}{2}+\bar{\theta}, \bar{\lambda}\right]$ stands for the pooling set that is the union of the set of $\lambda \in \Lambda$ and $\lambda \in\left[\frac{\delta}{2}+\bar{\theta}, \bar{\lambda}\right]$. The notation $\left[-\bar{\theta}, \frac{\delta}{2}+\bar{\theta}\right] \backslash \Lambda$ stands for the set of FIs that learn $\lambda \in\left[-\bar{\theta}, \frac{\delta}{2}+\bar{\theta}\right]$ but $\lambda \notin \Lambda$.
} 


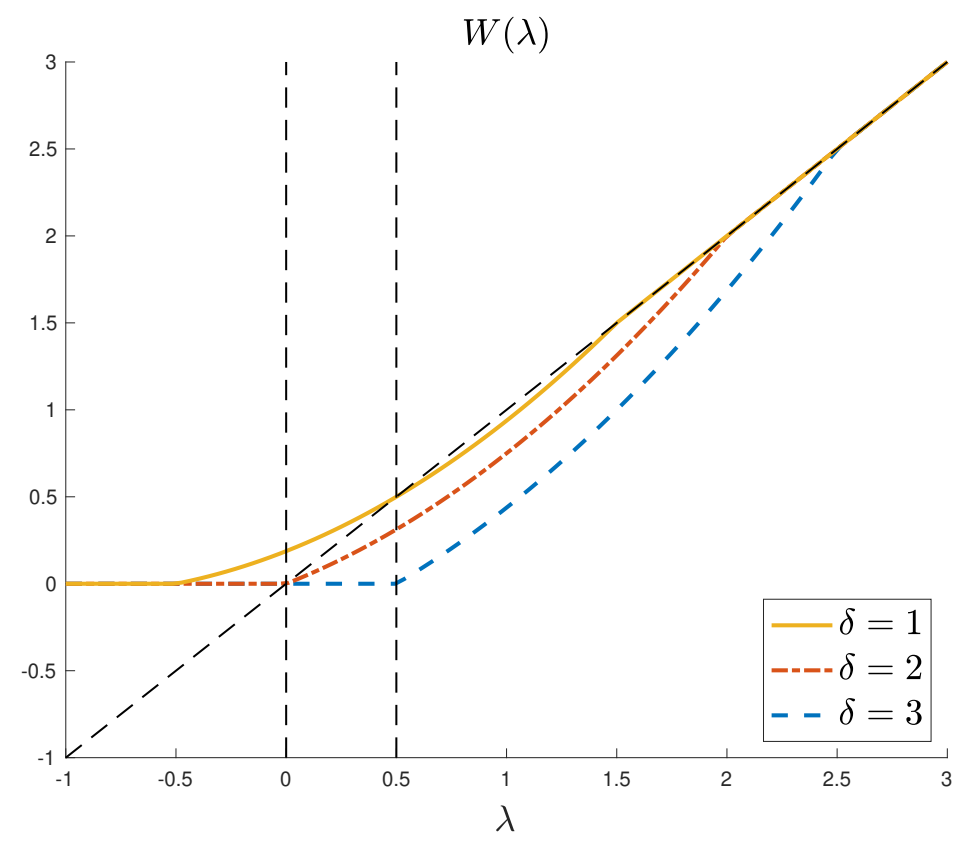

Figure 4: An illustration of Lemma 2. In this numerical example with $\bar{\theta}=1$, the colored lines represents $W(\lambda)$ evaluated at $\delta=1,2,3$, respectively, whereas the dashed 45-degree line represents $\lambda$. Observe that (1) when $\delta=1, \lambda \geq W(\lambda)$ if and only if $\lambda \geq 0.5$; (2) when $\delta=2$ or $3, \lambda \geq W(\lambda)$ if and only if $\lambda \geq 0$;

FI's project generates an expected surplus of

$$
W(\lambda) \equiv \frac{1}{2 \bar{\theta}} \int_{\frac{\delta}{2}-\lambda}^{\bar{\theta}}(\lambda+\theta) d \theta
$$

The objective function (8) reflects a non-trivial trade-off in the choice of the pooling set. Including a FI in the pooling set avoids runs on the FI but entails inefficient continuation if $\lambda+\theta<0$, while excluding a FI from the pooling set risks inefficient runs because, upon disclosure, investors withdraw excessively even if $\lambda+\theta>0$. A FI, therefore, should be added to the pooling set only when the expected surplus from the FI under pooling, $\lambda$, exceeds that under separating, $W(\lambda)$. The following lemma establishes an intuitive result that $\lambda \geq W(\lambda)$ only when the FI's early information $\lambda$ is above some threshold (as illustrated in Figure 4). 
Lemma $2 \lambda \geq W(\lambda)$ if and only if $\lambda \geq \lambda^{\sharp} \equiv \max \left\{0, \bar{\theta}-\frac{\delta}{2}\right\}$.

A direct implication of Lemma 2 is that, all FIs with $\lambda \geq \lambda^{\sharp}$ should be in the pooling set as long as this does not trigger runs on the pooled FIs (i.e., the constraint (9) does not bind when $\left.\Lambda=\left[\lambda^{\sharp}, \frac{\delta}{2}+\bar{\theta}\right]\right)$. Otherwise, the pooling set must be curtailed at a higher threshold for $\lambda$ to ensure the satisfaction of (9). Combining the two cases, we summarize the full characterization of the optimal reporting rule in the following proposition.

Proposition 4 The optimal reporting rule is that if $\lambda \geq \lambda^{*}$, the FI reports $x_{H}$ while if $\lambda<\lambda^{*}$, the FI reports $\lambda$ truthfully. There exists a unique cutoff $\hat{\delta}$ such that, the reporting threshold $\lambda^{*}$ satisfies:

1. if the liquidation cost $\delta<\hat{\delta}, \lambda^{*}=\lambda^{\sharp} \equiv \max \left\{0, \bar{\theta}-\frac{\delta}{2}\right\}$;

2. if $\delta \geq \hat{\delta}, \lambda^{*}$ is the unique solution to the equation $\mathbb{E}\left(\lambda \mid \lambda \geq \lambda^{*}\right)=\frac{\delta}{2}+\bar{\theta}$;

3. $\lambda^{*}$ decreases in $\delta$ if $\delta<\hat{\delta}$ and increases in $\delta$ if $\delta \geq \hat{\delta}$.

The first part of Proposition 4 states that the optimal reporting rule mandates full disclosure only when the FI receives some early information that is sufficiently unfavorable. Upon learning some favorable news, however, the FI should not recognize any changes in the accounting report. Note that this reporting rule resembles some aspects of an impairment rule in practice (e.g., the lower of cost or market (LCM) accounting rules, or the treatment of held-to-maturity debt securities), which recognizes unrealized losses but not unrealized gains. In this light, Proposition 4 lends some support to the practice of treating unrealized gains and losses asymmetrically on the grounds of financial stability. Compared to full disclosure, 


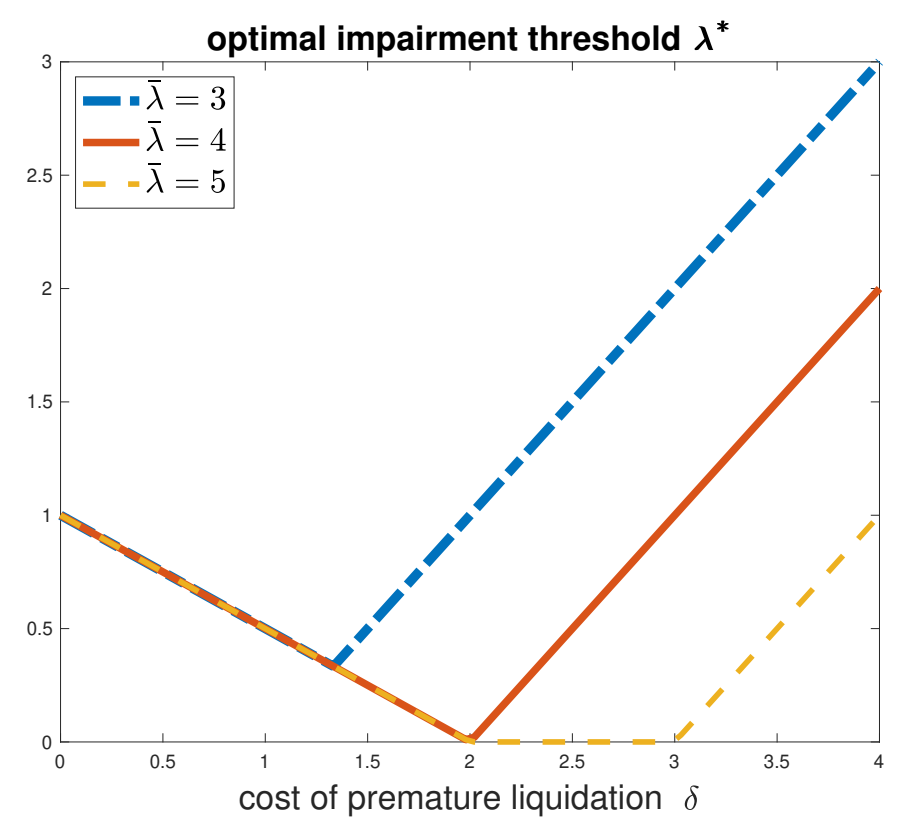

Figure 5: An illustration of Proposition 4. The figure shows that the reporting threshold $\lambda^{*}$ is U-shaped in the bank-run risk, captured by $\delta$, where both $\theta$ and $\lambda$ are uniformly distributed with $\bar{\theta}=1$ and $\bar{\lambda}=3,4,5$, correspondingly.

asymmetric disclosure rules like LCM result in higher surplus by achieving a better balance between inefficient runs and inefficient continuations. Under the optimal rule, some inefficient continuations are sustained among the set of FIs that are pooled but this is necessary to reduce inefficient runs.

The second part of Proposition 4 gives the optimal threshold below which the FI shall recognize an impairment. A key policy implication is that the impairment threshold should be tailored to FIs' vulnerability to the risk of runs (i.e., the cost of premature liquidation $\delta$ ). As illustrated in Figure 5, the exact relationship between the optimal impairment threshold and the risk of runs is in fact subtle and non-monotonic. More FIs should be allowed to recognize no impairment when the risk of runs rises but remains not too severe; however, when the risk of runs continues to increase and passes some critical level $\hat{\delta}$, the set of FIs 
that must report impairments should be expanded. The intuition is as follows. When the FI is relatively more resilient to runs (i.e., $\delta<\hat{\delta}$ ), it is easy to satisfy (9), i.e., the no-runon-pooled-FIs requirement. Absent the constraint, all FIs with $\lambda \geq \lambda^{\#}$ should be pooled together to minimize inefficient runs. As the run risk rises and FIs face more inefficient runs, the impairment threshold $\lambda^{*}=\lambda^{\#}$ should be lowered further in order to include more FIs in the pool. However, as the FI's exposure to bank runs becomes sufficiently large (i.e., $\delta \geq \hat{\delta}$ ), meeting (9) is no longer guaranteed. In other words, facing a significant run risk, one cannot reduce inefficient runs by simply pooling FIs together. This, in turn, requires some weaker FIs to recognize impairments in order to trim the pooling set. The higher the run risk, the harder to safeguard the pooled FIs from runs, and the more impairments that are needed.

\section{Extensions}

\subsection{General Distribution for $\theta$}

In the main model, we consider, for simplicity, a uniform distribution for the residual uncertainty $\theta$. To assess the robustness of our analysis, we examine an extension of our model in which $\theta$ has a general distribution $G($.$) and a density g($.$) with full support on [-\bar{\theta}, \bar{\theta}]$. Without loss of generality, we normalize the mean of $\theta$ to 0 . The following proposition characterizes a sufficient condition under which all the results in the main model extend qualitatively to the extension with a general distribution for $\theta$.

Proposition 5 If the density function of $\theta, g(\theta)$, satisfies $\frac{\partial \ln g(\theta)}{\partial \theta}<\frac{2}{\delta}$, the optimal reporting rule is an impairment rule as in Proposition 4. 
The condition $\frac{\partial \ln g(\theta)}{\partial \theta}<\frac{2}{\delta}$ implies that the density of $\theta$ should not increase too fast in $\theta$. For example, it is always met if $\theta$ follows a uniform distribution, the density of which is a constant. It is also met if $\theta$ follows a truncated normal distribution and the variance of $\theta$ is not too small, that is, the density of $\theta$ is relatively flat. ${ }^{11}$ The condition is needed to ensure that the FIs with moderate $\lambda \in\left[-\bar{\theta}, \bar{\theta}+\frac{\delta}{2}\right]$ should not be pooled among themselves (Part 3 of Proposition 2). To illustrate, consider, again, the two-FI example in Figure 3 and recall that pooling within the set $\lambda \in\left[-\bar{\theta}, \bar{\theta}+\frac{\delta}{2}\right]$ essentially replaces runs on the weak FI with runs on the strong one. When $\theta$ is uniformly distributed, the amount of runs on the weak FI reduced by pooling is the same as the amount of increases in the runs on the strong FI, that is,

$$
\operatorname{Pr}\left(\theta \in\left[\frac{\delta}{2}-\frac{\lambda_{1}+\lambda_{2}}{2}, \frac{\delta}{2}-\lambda_{2}\right)\right)=\operatorname{Pr}\left(\theta \in\left[\frac{\delta}{2}-\lambda_{1}, \frac{\delta}{2}-\frac{\lambda_{1}+\lambda_{2}}{2}\right)\right)=\frac{\lambda_{1}-\lambda_{2}}{4 \bar{\theta}}
$$

It is thus never optimal to exchange the same amount of runs on the weak FI for the same amount of runs on the strong FI. Given a general distribution of $\theta$, the changes of runs on the weak FI and on the strong FI can be different, depending on the distribution of $\theta$ across different values. When the density of $\theta$ is relatively flat in $\theta$, the magnitude of the reduced runs on the weak FI, i.e., $\operatorname{Pr}\left(\theta \in\left[\frac{\delta}{2}-\frac{\lambda_{1}+\lambda_{2}}{2}, \frac{\delta}{2}-\lambda_{2}\right)\right)$, does not dramatically exceed that of the increased runs on the strong FI, i.e., $\operatorname{Pr}\left(\theta \in\left[\frac{\delta}{2}-\lambda_{1}, \frac{\delta}{2}-\frac{\lambda_{1}+\lambda_{2}}{2}\right)\right)$, since there is enough probability at smaller values of $\theta \in\left[\frac{\delta}{2}-\lambda_{1}, \frac{\delta}{2}-\frac{\lambda_{1}+\lambda_{2}}{2}\right)$. As long as the amount of runs on strong FIs that pooling increases is not too small, it continues to be true that substituting

\footnotetext{
${ }^{11}$ More precisely, if $\tilde{\theta}$ is a normally distributed with mean 0 , variance $\sigma^{2}$ and truncated in the interval $[-\bar{\theta}, \bar{\theta}], \frac{\partial \ln g(\theta)}{\partial \theta}<\frac{2}{\delta}$ for all $\theta \in[-\bar{\theta}, \bar{\theta}]$ if and only if $\sigma>-\sqrt{\frac{\delta \bar{\theta}}{2}}$.
} 
runs on weak FIs with runs on strong ones is suboptimal, even when $\theta$ follows a general distribution.

\subsection{Noisy observation of $\theta$}

In the main model, we assume that investors observe $\theta$ (almost) perfectly. In this extension, we relax this assumption by allowing the precision of the investors' private information about $\theta$ to be less than infinity. For tractability, we assume the noise $\tilde{\varepsilon}_{i}$ in investor $i$ 's signal $s_{i}$ satisfies $\tilde{\varepsilon}_{i} \sim U[-\bar{\varepsilon}, \bar{\varepsilon}]$. Consistent with prior results in the global games literature (e.g., Morris and Shin, 2000; Liang and Zhang, 2019), we find that, when the precision of private information is sufficiently high, that is, when $\bar{\varepsilon}$ is sufficiently small, the equilibrium in the bankrun subgame is unique and all the results in our main setting hold qualitatively. We summarize this result in the following proposition.

Proposition 6 If the private information precision is sufficiently large, the optimal reporting rule is an impairment rule as in Proposition 4.

\section{Conclusion}

This paper examines the role of reporting rules in bank runs. We find that the optimal reporting rule mandates full disclosure of bad news but no disclosure otherwise. This rule is consistent with the impairment rule in current accounting standards, which requires adjusting the accounting value of assets only when the fair value falls below a certain cutoff. Our analysis shows that the optimality of the impairment rule lies in that it strikes a better balance between two types of inefficiency, i.e., inefficient panic-based runs on solvent financial 
institutions and inefficient continuations of insolvent ones, relative to a rule of full disclosure. The optimal reporting rule tolerates some inefficient continuations in exchange for fewer inefficient runs, and, in particular, seeks to eliminate any runs on financial institutions that do not recognize impairments.

Several important caveats regarding the optimality of the reporting rule we characterize are in order. We have only derived the optimal reporting rule within in a bank-run framework developed by Morris and Shin (2000). We employ this framework because it offers great analytical tractability for incorporating information disclosure, and has been widely adopted in the literature that studies information disclosure and bank runs. Employing the MorrisShin framework thus allows us to better compare our results with prior studies. Nonetheless, it should be noted that the Morris-Shin framework is a parsimonious model that does not capture all important aspects of bank runs, and there are other alternative plausible models of bank runs (e.g., Bryant, 1980; Diamond and Dybvig, 1983; Goldstein and Pauzer, 2005). Exploring the optimal reporting rule in other bank-run settings is an interesting avenue we leave for future research.

We have also only examined the ex ante optimal design of reporting rules and do not consider the implementation and the enforcement of the rules nor the reporting discretion by financial institutions. Gao and Jiang (2018) examine banks' discretionary reporting choices in the face of runs. An interesting next step is to combine the reporting discretion with the ex ante design of reporting rules, and analyze, given the reporting discretion, what the optimal reporting rule would be. ${ }^{12}$

\footnotetext{
${ }^{12}$ In spirit, such analysis is in line with Dye (2002), which examines the design of accounting standards considering manipulations by preparers of accounting reports.
} 


\section{References}

[1] Acharya, V., P. Schnabl, and G. Suarez. "Securitization Without Risk Transfer." Journal of Financial Economics 107 (2013): 515-536.

[2] Acharya, V., and S. Ryan. "Banks' financial reporting and financial system stability." Journal of Accounting Research 54, no. 2 (2016): 277-340.

[3] Anctil, R., J. Dickhaut, C. Kanodia, and B. Shapiro. "Information Transparency and Coordination Failure: Theory and Experiment." Journal of Accounting Research 42 (2004): 159-195.

[4] Anctil, R., J. Dickhaut, C. Johnson, and C. Kanodia. "Does Information Transparency Decrease Coordination Failure?" Games and Economic Behavior 70 (2010): 228-241.

[5] Armstrong, C., W. R. Guay, H. Mehran, and J. Weber. "The role of information and financial reporting in corporate governance: A review of the evidence and the implications for banking firms and the financial services industry." Economic Policy Review 22 (2016): 107-128.

[6] Beatty, A., and S. Liao. "Financial accounting in the banking industry: a review of the empirical literature." Journal of Accounting and Economics 58 (2014): 339-383.

[7] Bertomeu, J., and E. Cheynel. "Asset measurement in imperfect credit markets." Journal of Accounting Research 53, no. 5 (2015): 965-984.

[8] Bertomeu, J., L. Mahieux, and H. Sapra. "Interplay between accounting and prudential regulation." Available at SSRN 3266348 (2021). 
[9] Beyer, A., D. A. Cohen, T. Z. Lys, and B. R. Walther. "The Financial Reporting Environment: Review of the Recent Literature." Journal of Accounting and Economics 50, no. 2-3 (2010): 296-343.

[10] Bouvard, M., P. Chaigneau, and A. De Motta. "Transparency in the financial system: Rollover risk and crises." The Journal of Finance 70, no. 4 (2015): 1805-1837.

[11] Brunnermeier, M. "Deciphering the Liquidity and Credit Crunch 2007-08." Journal of Economic Perspective 23 (2009): 77-100.

[12] Bryant, J. "A model of reserves, bank runs, and deposit insurance." Journal of Banking and Finance 4, no. 4 (1980): 335-344.

[13] Cabrales, A., R. Nagel, and R. Armenter. "Equilibrium Selection through Incomplete Information in Coordination Games: an Experimental Study." Experimental Economics 10 (2007): 221-234.

[14] Corona, C., L. Nan, and G. Zhang. "Accounting Information Quality, Interbank Competition, and Bank Risk Taking." The Accounting Review 90 (2015): 967-985.

[15] Corona, C., L. Nan, and G. Zhang. "Banks' asset reporting frequency and capital regulation: An analysis of discretionary use of fair-value accounting." The Accounting Review 94, no. 2 (2019a): 157-178.

[16] Corona, C., L. Nan, and G. Zhang. "The Coordination Role of Stress Tests in Bank Risk Taking." Journal of Accounting Research 57, no. 5 (2019b): 1161-1200.

[17] Diamond, D., and P. Dybvig. "Bank Runs, Deposit Insurance, and Liquidity." Journal of Political Economy 91 (1983): 401-419. 
[18] Dye, R. A. "Classifications manipulation and Nash accounting standards." Journal of Accounting Research 40, no. 4 (2002): 1125-1162.

[19] Ewert, R., and A. Wagenhofer. "Earnings management, conservatism, and earnings quality." Foundations and Trends in Accounting 6, no. 2 (2012): 65-186.

[20] Financial Accounting Standards Board. U.S. GAAP Codification of Accounting Standards, Codification Topic 320: Investments-Debt and Equity Securities. Available at https://asc.fasb.org/imageRoot/34/116779634.pdf.

[21] Friedman, H., J. Hughes, and B. Michaeli. "Optimal reporting when additional information might arrive." Journal of Accounting and Economics 69, no. 2-3 (2020): 101276.

[22] Friedman, H., J. Hughes, and B. Michaeli. "A rationale for imperfect reporting standards." Management Science (2021) Forthcoming.

[23] Goldstein, I., and H. Sapra. "Should banks' stress test results be disclosed? an analysis of the costs and benefits." Foundations and Trends in Finance 8 (2014): 1-54.

[24] Gao, P., and X. Jiang. "Reporting Choices in the Shadow of Bank Runs." Journal of Accounting and Economics 65.1 (2018): 85-108.

[25] Goldstein, I., and Y. Leitner. "Stress tests and information disclosure." Journal of Economic Theory 177 (2018): 34-69.

[26] Goldstein, I., and A. Pauzner. "Demand-deposit contracts and the probability of bank runs." The Journal of Finance 60 (2005): 1293-1327.

[27] Heinemann, F., R. Nagel, and P. Ockenfels. "The Theory of Global Games on 
Test: Experimental Analysis of Coordination Games with Public and Private Information." Econometrica 72 (2004): 1583-1599.

[28] Huang, Z. "Optimal reporting systems with investor information acquisition." Working Paper (2019).

[29] Ijiri, Y. "Theory of accounting measurement." American Accounting Association (1975).

[30] Jiang, X., and M. Yang. "Properties of optimal accounting rules in a signaling game." Journal of Accounting and Economics 63, no. 2-3 (2017): 499-512.

[31] Jiang, X., and M. Yang. "Optimal Disclosure Rule, Private Benefits of Control and Efficient Liquidation." February 8, 2021. Available at SSRN: https://ssrn.com/abstract $=2857446$.

[32] Kamenica, E., and M. Gentzkow. "Bayesian persuasion." American Economic Review 101, no. 6 (2011): 2590-2615.

[33] Laux, C., and C. Leuz. "Did Fair-value Accounting Contribute to the Financial Crisis?." Journal of Economic Perspectives 24, no. 1 (2010): 93-118.

[34] Laux, V., and R. Zheng. "Early Warning Signals and Risk-Shifting Incentives." Working Paper (2021).

[35] Liang, P. J., and G. Zhang. "On the Social Value of Accounting Objectivity in Financial Stability." The Accounting Review 94, no 1 (2019): 229-248.

[36] Mahieux, L., H. Sapra, and G. Zhang. "CECL: Timely Loan Loss Provisioning and Bank Regulation.”Available at SSRN 3523321 (2020). 
[37] Morgan, D. "Rating Banks: Risk and Uncertainty in an Opaque Industry." The American Economic Review 92 (2002): 874-888.

[38] Morris, S., and H. Shin. "Rethinking Multiple Equilibria in Macroeconomic Modeling." Working paper, Princeton University (2000).

[39] Morris, S., and H. Shin. "Global Games: Theory and Applications." (2001).

[40] Shin, H. "Reflections on Northern Rock: The Bank Run that Heralded the Global Financial Crisis." Journal of Economic Perspective 23 (2009): 101-120.

[41] Stocken, P. C. "Strategic Accounting Disclosure." Foundations and Trends in Accounting 7, no. 4 (2013): 197-291.

[42] Goex, R. F., and A. Wagenhofer. "Optimal Impairment Rules." Journal of Accounting and Economics 48, no. 1 (2009): 2-16.

[43] Zhang, G. "Competition and Opacity in the Financial System." Management Science 67, no. 3 (2021): 1895-1913. 


\section{Appendix: proofs}

Proof. of Lemma 1: See the main text. We further provide explicit steps showing how we obtain (4) and (5). Since $\lambda$ and $\theta$ are independent to each other, we can rewrite $\mathbb{E}[\tilde{r}-\delta l \mid \hat{s}, x]$ as follows and obtain (4):

$$
\begin{aligned}
\mathbb{E}[\tilde{r}-\delta l \mid \hat{s}, x] & =\mathbb{E}[r \mid x, \hat{s}]-\delta \mathbb{E}[l \mid x, \hat{s}]=\mathbb{E}[\lambda+\theta \mid x, \hat{s}]-\delta \mathbb{E}[l \mid x, \hat{s}] \\
& =\mathbb{E}[\lambda \mid x, \hat{s}]+\mathbb{E}[\theta \mid x, \hat{s}]-\delta \mathbb{E}[l \mid x, \hat{s}] \\
& =\mathbb{E}[\lambda \mid x]+\mathbb{E}[\theta \mid \hat{s}]-\delta \mathbb{E}\left[\operatorname{Pr}\left(s_{j} \leqslant \hat{s}\right) \mid \hat{s}\right]
\end{aligned}
$$

where we substitute $l=\operatorname{Pr}\left(s_{j} \leqslant \hat{s}\right)$ in the last equality. We then can apply the law of iterated expectation to simplify $\mathbb{E}\left[\operatorname{Pr}\left(s_{j} \leqslant \hat{s}\right) \mid \hat{s}\right]$ and obtain $(5)$ :

$$
\begin{aligned}
\mathbb{E}\left[\operatorname{Pr}\left(s_{j} \leqslant \hat{s}\right) \mid \hat{s}\right] & =\mathbb{E}\left[\mathbb{E}\left[\operatorname{Pr}\left(s_{j} \leqslant \hat{s}\right) \mid \theta\right] \mid \hat{s}\right] \\
& =\mathbb{E}\left[\operatorname{Pr}\left(s_{j} \leqslant \hat{s} \mid \theta\right) \mid \hat{s}\right] \\
& =\mathbb{E}[\Phi(\sqrt{\beta}(\hat{s}-\theta)) \mid \hat{s}],
\end{aligned}
$$

where we use $\operatorname{Pr}\left(s_{j} \leqslant \hat{s} \mid \theta\right)=\Phi(\sqrt{\beta}(\hat{s}-\theta))$ in the last equality because $s_{i}=\tilde{\theta}+\tilde{\varepsilon}_{i}$ and $\tilde{\varepsilon}_{i} \sim N\left(0, \frac{1}{\beta}\right)$. Note that (4) and (5) do not depend on the distribution of $\theta$. When $\beta$ approaches infinity, the private signal $\hat{s}$ approaches $\theta$. Thus, $\mathbb{E}[\theta \mid \hat{s}]$ approaches $\theta$ and $\Phi(\sqrt{\beta}(\hat{s}-\theta))$ approaches $\frac{1}{2}$. The result then follows from (6) and $(7)$.

Proof. of Proposition 1: Note that under full disclosure (i.e., $x=\lambda$ ), $\mathbb{E}[\lambda \mid x]+\theta=\lambda+\theta$. Therefore, from Lemma 1, investors withdraw if and only if $\lambda+\theta<\frac{\delta}{2}$ and inefficient run occurs when $\lambda+\theta \in\left(0, \frac{\delta}{2}\right)$. We thus can state the equilibrium in the following cases: 
1. if $\lambda \geq \bar{\theta}+\frac{\delta}{2}$, we obtain $\lambda+\theta \geq \bar{\theta}+\frac{\delta}{2}+\theta \geq \bar{\theta}+\frac{\delta}{2}-\bar{\theta}=\frac{\delta}{2}>0$. Hence, no investors withdraw and the equilibrium is efficient;

2. if $\lambda \in\left[-\bar{\theta}+\frac{\delta}{2}, \bar{\theta}+\frac{\delta}{2}\right)$, investors withdraw when $\theta<-\lambda+\frac{\delta}{2}$ and inefficient run occurs when $\theta \in\left(-\lambda,-\lambda+\frac{\delta}{2}\right)$;

3. if $\lambda \in\left[-\bar{\theta},-\bar{\theta}+\frac{\delta}{2}\right)$, we obtain $\lambda+\theta<-\bar{\theta}+\frac{\delta}{2}+\theta<-\bar{\theta}+\frac{\delta}{2}+\bar{\theta}=\frac{\delta}{2}$ so that investors always withdraw and inefficient run occurs when $\theta \in(-\lambda, \bar{\theta})$;

4. if $\lambda<-\bar{\theta}$, we obtain $\lambda+\theta<-\bar{\theta}+\theta<-\bar{\theta}+\bar{\theta}=0$ so that investors always withdraw and the equilibrium is efficient.

Proof. of Proposition 2: The first two parts of the proposition are obvious so we focus on proving the last part. Denote by $W(\lambda)$ the expected social surplus when the FI discloses $\lambda$ perfectly. when $\lambda>\bar{\theta}+\frac{\delta}{2}$ and it is disclosed, the FI always survives such that the expected surplus is

$$
W(\lambda)=\lambda+\mathbb{E}[\theta]=\lambda .
$$

When $\lambda \in\left[-\bar{\theta}+\frac{\delta}{2}, \bar{\theta}+\frac{\delta}{2}\right]$ and it is disclosed, the FI survives if and only if it turns out $\theta>\frac{\delta}{2}-\lambda$. Thus, the expected surplus is

$$
W(\lambda)=\frac{1}{2 \bar{\theta}} \int_{\frac{\delta}{2}-\lambda}^{\bar{\theta}}(\lambda+\theta) d \theta=\frac{1}{4 \bar{\theta}}\left[(\bar{\theta}+\lambda)^{2}-\frac{\delta^{2}}{4}\right]
$$


with the first and second derivatives given by:

$$
W^{\prime}(\lambda)=\frac{\bar{\theta}+\lambda}{2 \bar{\theta}}
$$

and $W^{\prime \prime}(\lambda)=\frac{1}{2 \theta}>0$.

When $\lambda<-\bar{\theta}+\frac{\delta}{2}$ and it is disclosed, the investors always run and the expected surplus is $W(\lambda)=0$.

Note that $W(\lambda)$ is a convex function for $\lambda \in\left[-\bar{\theta}, \bar{\theta}+\frac{\delta}{2}\right]$. Furthermore, by the law of iterated expectation, the ex ante expected surplus of a FI who reports $x$ is equal to $W(\mathbb{E}(\lambda \mid x))$. The Jensen's inequality then implies that FIs with $\lambda \in\left[-\bar{\theta}, \bar{\theta}+\frac{\delta}{2}\right]$ shall never pool within themselves because

$$
\mathbb{E}[W(\lambda) \mid x]>W(\mathbb{E}(\lambda \mid x))
$$

Proof. of Proposition 3: Suppose that $\mathbb{E}\left(\lambda \mid x_{H}\right) \in\left(-\bar{\theta}, \bar{\theta}+\frac{\delta}{2}\right)$. We prove by contradiction that the total surplus can be improved by dividing those FIs reporting $x_{H}$ into two groups that report $x_{1}$ and $x_{2}$ correspondingly, such that, $\mathbb{E}\left(\lambda \mid x_{1}\right)=\bar{\theta}+\frac{\delta}{2}, \mathbb{E}\left(\lambda \mid x_{2}\right) \in\left(-\bar{\theta}, \bar{\theta}+\frac{\delta}{2}\right)$, and $x_{1}>x_{2}$. This statement is true by the Jensen's inequality because $W(\lambda)$ is a convex function for $\lambda \in\left[-\bar{\theta}, \bar{\theta}+\frac{\delta}{2}\right]$ as shown in the proof of Proposition 2.

Proof. of Lemma 2: From the main text, the expected surplus of a FI with $\lambda$ being pooled to survive is $\lambda+\mathbb{E}[\theta]=\lambda$. From Proposition 2, the expected surplus of being separated is $W(\lambda)$. Below we show that $\lambda \geq W(\lambda)$ if and only if $\lambda \geq \lambda^{\sharp}=\max \left\{0, \bar{\theta}-\frac{\delta}{2}\right\}$. 
First, when $\lambda>\bar{\theta}+\frac{\delta}{2}, W(\lambda)=\lambda$. When $\lambda \in\left[-\bar{\theta}+\frac{\delta}{2}, \bar{\theta}+\frac{\delta}{2}\right], \lambda \geq W(\lambda)$ is equivalent to

$$
\lambda-W(\lambda)=\frac{1}{2 \bar{\theta}} \int_{-\bar{\theta}}^{\frac{\delta}{2}-\lambda}(\lambda+\theta) d \theta=\frac{1}{4 \bar{\theta}}\left(\frac{\delta}{2}+\lambda-\bar{\theta}\right)\left(\frac{\delta}{2}-\lambda+\bar{\theta}\right) \geq 0
$$

which holds if and only if $\lambda \geq \bar{\theta}-\frac{\delta}{2}$. That is, $\lambda \geq W(\lambda)$ when $\lambda \geq \max \left\{-\bar{\theta}+\frac{\delta}{2}, \bar{\theta}-\frac{\delta}{2}\right\}$.

Second, when $\lambda<-\bar{\theta}+\frac{\delta}{2}$, it is easy to observe that $\lambda \geq W(\lambda)$ is equivalent to $\lambda \geq 0$. Therefore, $\lambda \geq W(\lambda)$ when $0 \leq \lambda \leq-\bar{\theta}+\frac{\delta}{2}$.

Combining the above two cases, we obtain that if $-\bar{\theta}+\frac{\delta}{2} \geq 0, \lambda \geq W(\lambda)$ when $\lambda \geq 0$; otherwise, if $-\bar{\theta}+\frac{\delta}{2}<0, \lambda \geq W(\lambda)$ when $\lambda \geq \bar{\theta}-\frac{\delta}{2}$. That is, $\lambda \geq W(\lambda)$ if and only if $\lambda \geq \lambda^{\sharp} \equiv \max \left\{0, \bar{\theta}-\frac{\delta}{2}\right\}$.

Proof. of Proposition 4: The proof proceeds in two parts. We first prove that the total surplus is maximized when those FIs with relatively higher $\lambda \mathrm{s}$, that is, $\lambda \in\left[\lambda^{*}, \frac{\delta}{2}+\bar{\theta}\right)$, are pooled with FIs $\lambda \geq \frac{\delta}{2}+\bar{\theta}$ (Part I). We then derive the comparative statics of $\lambda^{*}$ with respect to $\delta$ (Part II).

Part I: We consider two FIs with $\lambda_{1}$ and $\lambda_{2}$ such that $\lambda_{1}, \lambda_{2} \in\left[\lambda^{\sharp}, \bar{\theta}+\frac{\delta}{2}\right)$ and $\lambda_{1}>\lambda_{2}$. One reporting system $S_{1}$ pools a measure $\pi_{1}$ of $\lambda_{1}$ with a certain distribution of FIs in $\Lambda$ into reporting $x$, while the other reporting system $S_{2}$ pools a measure $\pi_{2}$ of $\lambda_{2}$ with the same distribution of FIs in $\Lambda$ into reporting $x$, such that $E\left(\lambda \mid x, S_{1}\right)=E\left(\lambda \mid x, S_{2}\right)=\bar{\theta}+\frac{\delta}{2}$, and all else equal. The conditions on $E(\lambda \mid x)$ imply

$$
\Pi \equiv \pi_{1}\left(\bar{\theta}+\frac{\delta}{2}-\lambda_{1}\right)=\pi_{2}\left(\bar{\theta}+\frac{\delta}{2}-\lambda_{2}\right)
$$


The surplus difference between $S_{1}$ and $S_{2}$ is determined by

$$
\pi_{1}\left(\lambda_{1}-W\left(\lambda_{1}\right)\right)-\pi_{2}\left(\lambda_{2}-W\left(\lambda_{2}\right)\right)
$$

Below we discuss two cases: $-\bar{\theta}+\frac{\delta}{2} \leq 0$ and $-\bar{\theta}+\frac{\delta}{2}>0$, to show the surplus is always higher with $S_{1}$ than with $S_{2}$.

First, if $-\bar{\theta}+\frac{\delta}{2} \leq 0$, we obtain $\lambda^{\sharp}=\max \left\{0, \bar{\theta}-\frac{\delta}{2}\right\}=\bar{\theta}-\frac{\delta}{2} \geq 0$ so $\lambda_{i} \in\left[\lambda^{\sharp}, \bar{\theta}+\frac{\delta}{2}\right] \subset$ $\left[-\bar{\theta}+\frac{\delta}{2}, \bar{\theta}+\frac{\delta}{2}\right]$. Moreover, for $\forall \lambda_{i} \in\left[-\bar{\theta}+\frac{\delta}{2}, \bar{\theta}+\frac{\delta}{2}\right]$, we obtain

$$
\begin{aligned}
\lambda_{i}-W\left(\lambda_{i}\right) & =\lambda_{i}-\frac{1}{2 \bar{\theta}} \int_{\frac{\delta}{2}-\lambda_{i}}^{\bar{\theta}}\left(\lambda_{i}+\theta\right) d \theta \\
& =\frac{1}{2 \bar{\theta}} \int_{-\bar{\theta}}^{\frac{\delta}{2}-\lambda_{i}}\left(\lambda_{i}+\theta\right) d \theta \\
& =\frac{1}{4 \bar{\theta}}\left(\bar{\theta}+\frac{\delta}{2}-\lambda_{i}\right)\left(-\bar{\theta}+\frac{\delta}{2}+\lambda_{i}\right) .
\end{aligned}
$$

The resulting surplus difference can be rewritten as

$$
\pi_{1}\left(\lambda_{1}-W\left(\lambda_{1}\right)\right)-\pi_{2}\left(\lambda_{2}-W\left(\lambda_{2}\right)\right)=\frac{1}{4 \theta} \Pi\left(\lambda_{1}-\lambda_{2}\right)>0
$$

Otherwise, if $-\bar{\theta}+\frac{\delta}{2}>0$, we obtain $\lambda^{\sharp}=\max \left\{0, \bar{\theta}-\frac{\delta}{2}\right\}=0$. Hence it is possible that $\lambda_{i} \in\left[\lambda^{\sharp},-\bar{\theta}+\frac{\delta}{2}\right]$ and in this case, $W\left(\lambda_{i}\right)=0$ so that $\lambda_{i}-W\left(\lambda_{i}\right)=\lambda_{i}$. There can be three possible scenarios:

1. If $\lambda_{1}, \lambda_{2} \in\left[-\bar{\theta}+\frac{\delta}{2}, \bar{\theta}+\frac{\delta}{2}\right]$, the surplus difference is $\frac{1}{4 \theta} \Pi\left(\lambda_{1}-\lambda_{2}\right)>0$. 
2. If $\bar{\theta}+\frac{\delta}{2}>\lambda_{1}>-\bar{\theta}+\frac{\delta}{2}>\lambda_{2} \geq \lambda^{\sharp}=0$, the surplus difference satisfies

$$
\pi_{1}\left(\lambda_{1}-W\left(\lambda_{1}\right)\right)-\pi_{2}\left(\lambda_{2}-W\left(\lambda_{2}\right)\right)>\frac{1}{4 \bar{\theta}} \Pi\left(\lambda_{1}-\lambda_{2}\right)>0
$$

The first inequality follows from that $W\left(\lambda_{2}\right)=0>\frac{1}{2 \theta} \int_{\frac{\delta}{2}-\lambda_{2}}^{\bar{\theta}}\left(\lambda_{2}+\theta\right) d \theta$ for $\lambda_{2} \in$ $\left[0,-\bar{\theta}+\frac{\delta}{2}\right]$

3. If $\lambda_{1}, \lambda_{2} \in\left[0,-\bar{\theta}+\frac{\delta}{2}\right]$, the surplus difference can be rewritten as

$$
\pi_{1}\left(\lambda_{1}-W\left(\lambda_{1}\right)\right)-\pi_{2}\left(\lambda_{2}-W\left(\lambda_{2}\right)\right)=\pi_{1} \lambda_{1}-\pi_{2} \lambda_{2}=\left(\bar{\theta}+\frac{\delta}{2}\right)\left(\pi_{1}-\pi_{2}\right)>0
$$

The last inequality holds because $\pi_{1}\left(\bar{\theta}+\frac{\delta}{2}-\lambda_{1}\right)=\pi_{2}\left(\bar{\theta}+\frac{\delta}{2}-\lambda_{2}\right)$ and $\bar{\theta}+\frac{\delta}{2}>\lambda_{1}>$ $\lambda_{2}$, such that $\pi_{1}>\pi_{2}$.

In sum, the surplus improves if one prioritizes pooling a FI with higher $\lambda$. Hence, the surplus is maximized when those FIs with $\lambda \in\left[\lambda^{*}, \frac{\delta}{2}+\bar{\theta}\right)$ are pooled with FIs $\lambda \geq \frac{\delta}{2}+\bar{\theta}$. The objective function (8) and the constraint (9) can be simplified to

$$
\begin{aligned}
& \max _{\lambda^{*} \in\left[-\bar{\theta}, \frac{\delta}{2}+\bar{\theta}\right]} \int_{\lambda^{*}}^{\bar{\lambda}} \lambda f(\lambda) d \lambda+\int_{-\bar{\theta}}^{\lambda^{*}} W(\lambda) f(\lambda) d \lambda, \\
& \text { s.t., } \mathbb{E}\left(\lambda \mid \lambda \geq \lambda^{*}\right) \geq \frac{\delta}{2}+\bar{\theta} .
\end{aligned}
$$

It is then straight forward to show that the optimal threshold satisfies $\lambda^{*}=\max \left\{\lambda^{\sharp}, \tau^{*}\right\}$, where we denote $\tau^{*}$ the solution to the equation $\mathbb{E}\left(\lambda \mid \lambda \geq \tau^{*}\right)=\frac{\delta}{2}+\bar{\theta}$.

Part II: We now derive the comparative statics of $\lambda^{*}$ in $\delta$. First, when $\delta \rightarrow 0$, we have $\lambda^{\sharp}=\max \left\{0, \bar{\theta}-\frac{\delta}{2}\right\}=\bar{\theta}-\frac{\delta}{2} \rightarrow \bar{\theta}$, and $\tau^{*} \rightarrow \tau_{0}$ where $\tau_{0}<\bar{\theta}$ is determined by 
$\mathbb{E}\left(\lambda \mid \lambda \geq \tau_{0}\right)=\bar{\theta}$. Hence, as $\lambda^{*}=\max \left\{\lambda^{\sharp}, \tau^{*}\right\}$, we obtain

$$
\lim _{\delta \rightarrow 0} \lambda^{*}=\max \left\{\bar{\theta}, \tau_{0}\right\}=\bar{\theta} .
$$

It is easy to see that $\lambda^{\sharp}$ decreases in $\delta$ while $\tau^{*}$ increases in $\delta$. As $\delta$ increases from $0, \lambda^{\sharp}$ decreases from $\bar{\theta}$ while $\tau^{*}$ increases from $\tau_{0}$. Note that when $\delta$ increases to $2 \bar{\theta}, \lambda^{\sharp}$ decreases to 0 while $\tau^{*}$ increases to $\tau_{1}$, where $\tau_{1}$ is determined by $\mathbb{E}\left(\lambda \mid \lambda \geq \tau_{1}\right)=2 \bar{\theta}$. There can be two cases depending on whether $\tau_{1}>0$ :

1. if $\tau_{1}>0$, there exists a unique $\delta_{0} \in(0,2 \bar{\theta})$ such that $\lambda^{\sharp} \geq \tau^{*}$ if and only if $\delta \in\left(0, \delta_{0}\right]$. Hence, we obtain

$$
\lambda^{*}=\left\{\begin{array}{ll}
\lambda^{\sharp} & \text { if } \delta \leq \delta_{0} \\
\tau^{*} & \text { if } \delta>\delta_{0}
\end{array},\right.
$$

where $\lambda^{*}$ first decreases in $\delta$ when $\delta \leq \delta_{0}$ and then increases in $\delta$ when $\delta>\delta_{0}$.

2. if $\tau_{1} \leq 0$, there exists a unique $\delta_{1}>\delta_{0}$ such that $\tau^{*} \geq 0$ if and only if $\delta \geq \delta_{1} \equiv$ $2(\mathbb{E}(\lambda \mid \lambda \geq 0)-\bar{\theta})$. Hence, we obtain

$$
\lambda^{*}=\left\{\begin{array}{ll}
\lambda^{\sharp} & \text { if } \delta \leq \delta_{1} \\
\tau^{*} & \text { if } \delta>\delta_{1}
\end{array},\right.
$$

where $\lambda^{*}$ first decreases in $\delta$ when $\delta \leq \delta_{0}$, then stays at 0 when $\delta \in\left[\delta_{0}, \delta_{1}\right]$, and then increases in $\delta$ when $\delta>\delta_{1}$. 
Proof. of Proposition 5: The proof proceeds in two steps. First, we provide in Lemma 3 a necessary and sufficient condition for $W(\lambda)$ to be convex in the region of $\lambda \in\left[-\bar{\theta}, \bar{\theta}+\frac{\delta}{2}\right]$. Second, we show that if $W(\lambda)$ is convex for $\lambda \in\left[-\bar{\theta}, \bar{\theta}+\frac{\delta}{2}\right]$, all our main results follow (using Lemma 4 and 5).

Lemma $3 W(\lambda)$ is a convex function for $\lambda \in\left[-\bar{\theta}, \bar{\theta}+\frac{\delta}{2}\right]$ if and only if $\frac{g^{\prime}(\theta)}{g(\theta)}<\frac{2}{\delta}$ for $\theta \in$ $[-\bar{\theta}, \bar{\theta}]$

Proof. When $\lambda \in\left[-\bar{\theta}+\frac{\delta}{2}, \bar{\theta}+\frac{\delta}{2}\right]$ is disclosed, the FI survives if and only if it turns out $\theta>\frac{\delta}{2}-\lambda$. Thus, the expected surplus given $\lambda$ is

$$
W(\lambda)=\int_{\frac{\delta}{2}-\lambda}^{\bar{\theta}}(\lambda+\theta) g(\theta) d \theta
$$

with the first and the second derivatives:

$$
\begin{aligned}
W^{\prime}(\lambda) & =\int_{\frac{\delta}{2}-\lambda}^{\bar{\theta}} g(\theta) d \theta+\left(\lambda+\left(\frac{\delta}{2}-\lambda\right)\right) g\left(\frac{\delta}{2}-\lambda\right) \\
& =\int_{\frac{\delta}{2}-\lambda}^{\bar{\theta}} g(\theta) d \theta+\frac{\delta}{2} g\left(\frac{\delta}{2}-\lambda\right)>0,
\end{aligned}
$$

and

$$
W^{\prime \prime}(\lambda)=g\left(\frac{\delta}{2}-\lambda\right)-\frac{\delta}{2} g^{\prime}\left(\frac{\delta}{2}-\lambda\right)
$$

which is positive if and only if $\frac{g^{\prime}\left(\frac{\delta}{2}-\lambda\right)}{g\left(\frac{\delta}{2}-\lambda\right)}<\frac{2}{\delta}$. When $\lambda<-\bar{\theta}+\frac{\delta}{2}$ is disclosed, the FI always suffers runs and the expected surplus is $W(\lambda)=0$. Hence, $W(\lambda)$ is a convex function for $\lambda \in\left[-\bar{\theta}, \bar{\theta}+\frac{\delta}{2}\right]$ if and only if $\frac{g^{\prime}(\theta)}{g(\theta)}<\frac{2}{\delta}$ for $\forall \theta \in[-\bar{\theta}, \bar{\theta}]$. Q.E.D. 
Lemma 4 When $W(\lambda)$ is a convex function for $\lambda \in\left[-\bar{\theta}, \bar{\theta}+\frac{\delta}{2}\right]$, there exists a unique threshold $\lambda^{\sharp}$ such that $\lambda \geq W(\lambda)$ if and only if $\lambda \geq \lambda^{\sharp}$. Moreover, $\lambda^{\sharp} \in\left(0, \bar{\theta}+\frac{\delta}{2}\right)$ if and only if $-\bar{\theta}+\frac{\delta}{2}<0$ and $\lambda^{\sharp}=0$ otherwise.

Proof. Denote $\Delta(\lambda) \equiv W(\lambda)-\lambda$. It is equivalent to show that $\Delta(\lambda) \leq 0$ if and only if $\lambda \geq \lambda^{\sharp}$. Obviously, when $\lambda \geq \bar{\theta}+\frac{\delta}{2}$, we obtain $W(\lambda)=\lambda$ and thus $\Delta(\lambda)=0$. It is easy to show that $\Delta^{\prime \prime}(\lambda)=W^{\prime \prime}(\lambda)$, so $\Delta(\lambda)$ is also a convex function for $\lambda \in\left[-\bar{\theta}, \bar{\theta}+\frac{\delta}{2}\right]$. Moreover, we obtain

$$
\begin{aligned}
\Delta^{\prime}\left(\bar{\theta}+\frac{\delta}{2}\right) & =W^{\prime}\left(\bar{\theta}+\frac{\delta}{2}\right)-1 \\
& =\int_{-\bar{\theta}}^{\bar{\theta}} g(\theta) d \theta+\frac{\delta}{2} g(-\bar{\theta})-1 \\
& =\frac{\delta}{2} g(-\bar{\theta})>0 .
\end{aligned}
$$

To further find the region in which $\Delta(\lambda) \leq 0$ (that is, $\lambda \geq W(\lambda)$ ), we discuss the following two cases:

1. If $-\bar{\theta}+\frac{\delta}{2} \geq 0, \Delta(\lambda) \leq 0$ if and only if $\lambda \geq 0$. This is because:

$$
\Delta\left(-\bar{\theta}+\frac{\delta}{2}\right)=W\left(-\bar{\theta}+\frac{\delta}{2}\right)-\left(-\bar{\theta}+\frac{\delta}{2}\right)=-\left(-\bar{\theta}+\frac{\delta}{2}\right) \leq 0
$$


and by the convexity of $\Delta(\lambda)$, for $\forall \lambda \in\left(-\bar{\theta}+\frac{\delta}{2}, \bar{\theta}+\frac{\delta}{2}\right)$, the following holds:

$$
\begin{aligned}
\Delta(\lambda) & =\Delta\left(\frac{\bar{\theta}+\frac{\delta}{2}-\lambda}{2 \bar{\theta}}\left(-\bar{\theta}+\frac{\delta}{2}\right)+\frac{\lambda-\left(-\bar{\theta}+\frac{\delta}{2}\right)}{2 \bar{\theta}}\left(\bar{\theta}+\frac{\delta}{2}\right)\right) \\
& \leq \frac{\bar{\theta}+\frac{\delta}{2}-\lambda}{2 \bar{\theta}} \Delta\left(-\bar{\theta}+\frac{\delta}{2}\right)+\frac{\lambda-\left(-\bar{\theta}+\frac{\delta}{2}\right)}{2 \bar{\theta}} \Delta\left(\bar{\theta}+\frac{\delta}{2}\right) \\
& =\frac{\bar{\theta}+\frac{\delta}{2}-\lambda}{2 \bar{\theta}} \Delta\left(-\bar{\theta}+\frac{\delta}{2}\right) \leq 0 .
\end{aligned}
$$

Moreover, if $\lambda \leq-\bar{\theta}+\frac{\delta}{2}$, we obtain $W(\lambda)=0$ and thus $\Delta(\lambda)=-\lambda$, which is nonpositive if and only if $\lambda \geq 0$.

2. If $-\bar{\theta}+\frac{\delta}{2}<0$, there is a unique solution $\lambda^{+} \in\left(0, \bar{\theta}+\frac{\delta}{2}\right)$ satisfying $\Delta\left(\lambda^{+}\right)=0$, and $\Delta(\lambda) \leq 0$ if and only if $\lambda \geq \lambda^{+}$. Since $W(\lambda)$ is increasing for $\lambda \in\left[-\bar{\theta}+\frac{\delta}{2}, \bar{\theta}+\frac{\delta}{2}\right]$, we obtain $\forall \lambda \in\left(-\bar{\theta}+\frac{\delta}{2}, 0\right], W(\lambda)>W\left(-\bar{\theta}+\frac{\delta}{2}\right)=0$ and hence,

$$
\Delta(\lambda)=W(\lambda)-\lambda>0
$$

Due to the convexity of $\Delta(\lambda)$, there can be at most two solutions to $\Delta(\lambda)=0$ for $\lambda \in\left[-\bar{\theta}+\frac{\delta}{2}, \bar{\theta}+\frac{\delta}{2}\right]$. One of the solutions is $\lambda=\bar{\theta}+\frac{\delta}{2}$ as $\Delta\left(\bar{\theta}+\frac{\delta}{2}\right)=0$. We have shown that $\Delta^{\prime}\left(\bar{\theta}+\frac{\delta}{2}\right)>0$, which suggests that there exists a small enough $\epsilon>0$ such that $\Delta\left(\bar{\theta}+\frac{\delta}{2}-\epsilon\right)<0$. Combining with that $\Delta(0)>0$, we show that the other solution denoted by $\lambda^{+}$exists and satisfies that $\lambda^{+} \in\left(0, \bar{\theta}+\frac{\delta}{2}\right)$. In other words, when $\lambda$ increases from 0 to $\bar{\theta}+\frac{\delta}{2}, \Delta(\lambda)$ first decreases from above zero to below zero after hitting zero at $\lambda=\lambda^{+}$, then starts increasing from below zero until hitting zero at $\lambda=\bar{\theta}+\frac{\delta}{2}$. This implies that $\Delta(\lambda)<0$ for $\lambda \in\left(\lambda^{+}, \bar{\theta}+\frac{\delta}{2}\right)$, and $\Delta(\lambda)>0$ 
for $\lambda \in\left(0, \lambda^{+}\right)$. Meanwhile, if $\lambda \leq-\bar{\theta}+\frac{\delta}{2}<0$, we obtain $W(\lambda)=0$ and thus $\Delta(\lambda)=-\lambda \geq-\left(-\bar{\theta}+\frac{\delta}{2}\right)>0$.

The result follows after combining the above two cases. Q.E.D.

Lemma 5 When $W(\lambda)$ is a convex function for $\lambda \in\left[-\bar{\theta}, \bar{\theta}+\frac{\delta}{2}\right]$, the total surplus is maximized when those FIs with relatively higher $\lambda$ s, that is, $\lambda \in\left[\lambda^{*}, \frac{\delta}{2}+\bar{\theta}\right)$, are pooled with $F I s \lambda \geq \frac{\delta}{2}+\bar{\theta}$.

Proof. Consider two FIs with $\lambda_{1}$ and $\lambda_{2}$ such that $\lambda_{1}, \lambda_{2} \in\left[\lambda^{\sharp}, \bar{\theta}+\frac{\delta}{2}\right)$ and $\lambda_{1}>\lambda_{2}$. One reporting system $S_{1}$ pools a measure $\pi_{1}$ of $\lambda_{1}$ with a certain distribution of FIs in $\Lambda$ into reporting $x$, while the other reporting system $S_{2}$ pools a measure $\pi_{2}$ of $\lambda_{2}$ with the same distribution of FIs in $\Lambda$ into reporting $x$, such that $\mathbb{E}\left(\lambda \mid x, S_{1}\right)=\mathbb{E}\left(\lambda \mid x, S_{2}\right)=\bar{\theta}+\frac{\delta}{2}$, and all else equal. The conditions on $\mathbb{E}(\lambda \mid x)$ imply

$$
\Pi \equiv \pi_{1}\left(\bar{\theta}+\frac{\delta}{2}-\lambda_{1}\right)=\pi_{2}\left(\bar{\theta}+\frac{\delta}{2}-\lambda_{2}\right)
$$

The surplus difference between $S_{1}$ and $S_{2}$ is determined by

$$
\pi_{1}\left(\lambda_{1}-W\left(\lambda_{1}\right)\right)-\pi_{2}\left(\lambda_{2}-W\left(\lambda_{2}\right)\right)
$$


Due to the conditions on $\mathbb{E}(\lambda \mid x)$ and $W\left(\bar{\theta}+\frac{\delta}{2}\right)=\bar{\theta}+\frac{\delta}{2}$, it is equivalent to show that

$$
\begin{aligned}
& \Pi \frac{\left(\lambda_{1}-W\left(\lambda_{1}\right)\right)}{\bar{\theta}+\frac{\delta}{2}-\lambda_{1}}>\Pi \frac{\left(\lambda_{2}-W\left(\lambda_{2}\right)\right)}{\bar{\theta}+\frac{\delta}{2}-\lambda_{2}} \\
\Leftrightarrow & \frac{\left(\lambda_{1}-W\left(\lambda_{1}\right)\right)}{\bar{\theta}+\frac{\delta}{2}-\lambda_{1}}+1>\frac{\left(\lambda_{2}-W\left(\lambda_{2}\right)\right)}{\bar{\theta}+\frac{\delta}{2}-\lambda_{2}}+1 \\
\Leftrightarrow & \frac{\left(\bar{\theta}+\frac{\delta}{2}-W\left(\lambda_{1}\right)\right)}{\bar{\theta}+\frac{\delta}{2}-\lambda_{1}}>\frac{\left(\bar{\theta}+\frac{\delta}{2}-W\left(\lambda_{2}\right)\right)}{\bar{\theta}+\frac{\delta}{2}-\lambda_{2}} \\
\Leftrightarrow & \frac{W\left(\bar{\theta}+\frac{\delta}{2}\right)-W\left(\lambda_{1}\right)}{\bar{\theta}+\frac{\delta}{2}-\lambda_{1}}>\frac{W\left(\bar{\theta}+\frac{\delta}{2}\right)-W\left(\lambda_{2}\right)}{\bar{\theta}+\frac{\delta}{2}-\lambda_{2}} \\
\Leftrightarrow & \frac{\int_{\lambda_{1}}^{\bar{\theta}+\frac{\delta}{2}} W^{\prime}(\lambda) d \lambda}{\bar{\theta}+\frac{\delta}{2}-\lambda_{1}}>\frac{\int_{\lambda_{2}}^{\bar{\theta}+\frac{\delta}{2}} W^{\prime}(\lambda) d \lambda}{\bar{\theta}+\frac{\delta}{2}-\lambda_{2}} \\
\Leftrightarrow & \frac{\int_{\lambda_{1}}^{\bar{\theta}+\frac{\delta}{2}} W^{\prime}(\lambda) d \lambda}{\bar{\theta}+\frac{\delta}{2}-\lambda_{1}}>\frac{\int_{\lambda_{2}}^{\lambda_{1}} W^{\prime}(\lambda) d \lambda}{\lambda_{1}-\lambda_{2}},
\end{aligned}
$$

which is true for $\forall \lambda_{1}, \lambda_{2} \in\left[-\bar{\theta}, \bar{\theta}+\frac{\delta}{2}\right)$ with $\lambda_{1}>\lambda_{2}$, because $W^{\prime}(\lambda)>0, W^{\prime \prime}(\lambda)>0$ for $\lambda \in\left[-\bar{\theta}+\frac{\delta}{2}, \bar{\theta}+\frac{\delta}{2}\right]$ and $W(\lambda)=0, W^{\prime}(\lambda)=0$ for $\lambda \in\left[-\bar{\theta},-\bar{\theta}+\frac{\delta}{2}\right]$

In sum, the surplus improves if one prioritizes pooling a FI with higher $\lambda$. Hence, the surplus is maximized when those FIs with $\lambda \in\left[\lambda^{*}, \frac{\delta}{2}+\bar{\theta}\right)$ are pooled with FIs $\lambda \geq \frac{\delta}{2}+\bar{\theta}$.

\section{Q.E.D.}

Note that with Lemma 4 and 5, Proposition 4 can be proved following similar steps in the proof of Proposition 4.

Proof. of Proposition 6: As shown in Section 3.1, the investor who observes a $s_{i}$ equal to $\hat{s}$ is indifferent between staying and withdrawing in equilibrium, which in turn gives:

$$
\mathbb{E}[\lambda \mid x]+\mathbb{E}\left[\theta-\delta \operatorname{Pr}\left(s_{j}<\hat{s}\right) \mid \hat{s}\right]=0
$$


When $\bar{\varepsilon}$ is small, we can show that

$$
\mathbb{E}\left[\theta-\delta \operatorname{Pr}\left(s_{j}<\hat{s}\right) \mid \hat{s}\right]=\left\{\begin{array}{ll}
\bar{\theta}-\delta & \text { if } \hat{s} \geq \bar{\theta}+\bar{\varepsilon} \\
\frac{\hat{s}+\bar{\theta}-\bar{\varepsilon}}{2}-\delta\left(\frac{1}{2}+\frac{\hat{s}-\bar{\theta}+\bar{\varepsilon}}{4 \bar{\varepsilon}}\right) & \text { if } \hat{s} \in(\bar{\theta}-\bar{\varepsilon}, \bar{\theta}+\bar{\varepsilon}) \\
\hat{s}-\frac{\delta}{2} & \text { if } \hat{s} \in[-\bar{\theta}+\bar{\varepsilon}, \bar{\theta}-\bar{\varepsilon}] \\
\frac{\hat{s}-\bar{\theta}+\bar{\varepsilon}}{2}-\delta \frac{\hat{s}+\bar{\theta}+\bar{\varepsilon}}{4 \bar{\varepsilon}} & \text { if } \hat{s} \in(-\bar{\theta}-\bar{\varepsilon},-\bar{\theta}+\bar{\varepsilon}) \\
-\bar{\theta} & \text { if } \hat{s} \leq-\bar{\theta}-\bar{\varepsilon}
\end{array},\right.
$$

and $\mathbb{E}\left[\theta-\delta \operatorname{Pr}\left(s_{j}<\hat{s}\right) \mid \hat{s}\right]$ increases in $\hat{s}$ when $\hat{s} \in[-\bar{\theta}+\bar{\varepsilon}, \bar{\theta}-\bar{\varepsilon}]$, while decreasing in $\hat{s}$ when either $\hat{s} \in(\bar{\theta}-\bar{\varepsilon}, \bar{\theta}+\bar{\varepsilon})$ or $\hat{s} \in(-\bar{\theta}-\bar{\varepsilon},-\bar{\theta}+\bar{\varepsilon})$. Hence, when $\mathbb{E}[\lambda \mid x]<-\bar{\theta}+\bar{\varepsilon}+\frac{\delta}{2}$, we obtain $\mathbb{E}[\lambda \mid x]+\mathbb{E}\left[\theta-\delta \operatorname{Pr}\left(s_{j}<\hat{s}\right) \mid \hat{s}\right]<0$ and all investors withdraw, whereas when $\mathbb{E}[\lambda \mid x]>\bar{\theta}-\bar{\varepsilon}+\frac{\delta}{2}$, we obtain $\mathbb{E}[\lambda \mid x]+\mathbb{E}\left[\theta-\delta \operatorname{Pr}\left(s_{j}<\hat{s}\right) \mid \hat{s}\right]>0$ and all investors choose not to withdraw. When $\mathbb{E}[\lambda \mid x] \in\left[-\bar{\theta}+\bar{\varepsilon}+\frac{\delta}{2}, \bar{\theta}-\bar{\varepsilon}+\frac{\delta}{2}\right]$, the threshold $\hat{s}$ is determined by $\mathbb{E}[\lambda \mid x]+\hat{s}-\frac{\delta}{2}=0$ in the stable equilibrium, such that any investor with a signal $s_{i} \leq \hat{s}=\frac{\delta}{2}-\mathbb{E}[\lambda \mid x]$ withdraws and other investors stay. In this case, if $\theta \leq \frac{\delta}{2}-\mathbb{E}[\lambda \mid x]-\bar{\varepsilon}$ all investors withdraw; if $\theta \in\left(\frac{\delta}{2}-\mathbb{E}[\lambda \mid x]-\bar{\varepsilon}, \frac{\delta}{2}-\mathbb{E}[\lambda \mid x]+\bar{\varepsilon}\right)$, a measure of $\frac{\frac{\delta}{2}-\mathbb{E}[\lambda \mid x]+\bar{\varepsilon}-\theta}{2 \bar{\varepsilon}}$ of investors withdraw; and if $\theta \geq \frac{\delta}{2}-\mathbb{E}[\lambda \mid x]+\bar{\varepsilon}$, all investors stay.

In sum, an investor $i$ withdraws from the FI in equilibrium if and only if one of the following conditions is satisfied:

1. $\mathbb{E}[\lambda \mid x]<-\bar{\theta}+\bar{\varepsilon}+\frac{\delta}{2}$

2. $\mathbb{E}[\lambda \mid x] \in\left[-\bar{\theta}+\bar{\varepsilon}+\frac{\delta}{2}, \bar{\theta}-\bar{\varepsilon}+\frac{\delta}{2}\right]$ and $s_{i} \leq \frac{\delta}{2}-\mathbb{E}[\lambda \mid x]$.

Similarly, we denote by $W(\lambda)$ the expected social surplus given a FI with $\lambda$ being perfectly 
disclosed. When $\lambda>\bar{\theta}-\bar{\varepsilon}+\frac{\delta}{2}$ is perfectly disclosed, no investor withdraws and the FI always survives so the expected surplus is

$$
W(\lambda)=\lambda+\mathbb{E}[\theta]=\lambda
$$

When $\lambda \in\left[-\bar{\theta}+\bar{\varepsilon}+\frac{\delta}{2}, \bar{\theta}-\bar{\varepsilon}+\frac{\delta}{2}\right]$ is disclosed, investors who observe a signal $s_{i} \leq \frac{\delta}{2}-\lambda$ withdraws. Thus, the expected surplus is

$$
\begin{aligned}
W(\lambda) & =\frac{1}{2 \bar{\theta}}\left[\int_{\frac{\delta}{2}-\lambda-\bar{\varepsilon}}^{\frac{\delta}{2}-\lambda+\bar{\varepsilon}} \frac{\lambda+\theta+\bar{\varepsilon}-\frac{\delta}{2}}{2 \bar{\varepsilon}}\left(\lambda+\theta-\delta \frac{\frac{\delta}{2}-\lambda+\bar{\varepsilon}-\theta}{2 \bar{\varepsilon}}\right) d \theta+\int_{\frac{\delta}{2}-\lambda+\bar{\varepsilon}}^{\bar{\theta}}(\lambda+\theta) d \theta\right] \\
& =\frac{1}{2 \bar{\theta}} \int_{\frac{\delta}{2}-\bar{\varepsilon}}^{\frac{\delta}{2}+\bar{\varepsilon}} \frac{r+\bar{\varepsilon}-\frac{\delta}{2}}{2 \bar{\varepsilon}}\left(r-\delta \frac{\frac{\delta}{2}+\bar{\varepsilon}-r}{2 \bar{\varepsilon}}\right) d r+\frac{1}{4 \bar{\theta}}\left[(\bar{\theta}+\lambda)^{2}-\left(\frac{\delta}{2}+\bar{\varepsilon}\right)^{2}\right]
\end{aligned}
$$

which satisfies

$$
W^{\prime}(\lambda)=\frac{\bar{\theta}+\lambda}{2 \bar{\theta}}
$$

and $W^{\prime \prime}(\lambda)=\frac{1}{2 \theta}>0$.

When $\lambda<-\bar{\theta}+\bar{\varepsilon}+\frac{\delta}{2}$ is disclosed, the FI always suffers runs and the expected surplus is $W(\lambda)=0$.

Hence, $W(\lambda)$ is a convex function for $\lambda \in\left[-\bar{\theta}, \bar{\theta}-\bar{\varepsilon}+\frac{\delta}{2}\right]$. The results then follow using proofs similar to the proof of Proposition 5. 